\title{
Relative concentration and structure of native defects in GaP
}

\author{
A. Höglund, ${ }^{1}$ C. W. M. Castleton, ${ }^{2,3}$ and S. Mirbt ${ }^{1}$ \\ ${ }^{1}$ Department of Physics, Uppsala University, Box 530, SE-75121, Uppsala, Sweden \\ ${ }^{2}$ Materials and Semiconductor Physics Laboratory, Royal Institute of Technology (KTH), Electrum 229, SE-16440 Kista, Sweden \\ ${ }^{3}$ Department of Materials Chemistry, Box 538, SE-75121, Uppsala, Sweden
}

(Received 22 November 2004; revised manuscript received 12 August 2005; published 30 November 2005)

\begin{abstract}
The native defects in the compound semiconductor $\mathrm{GaP}$ have been studied using a pseudopotential density functional theory method in order to determine their relative concentrations and the most stable charge states. The electronic and atomic structures are presented and the defect concentrations are estimated using calculated formation energies. Relaxation effects are taken into account fully and produce negative- $U$ charge transfer levels for $\mathrm{V}_{\mathrm{P}}$ and $\mathrm{P}_{\mathrm{Ga}}$. The concentration of $\mathrm{V}_{\mathrm{Ga}}$ is in good agreement with the results of positron annihilation experiments. The charge transfer levels presented compare qualitatively well with experiments where available. The effect of stoichiometry on the defect concentrations is also described and is shown to be considerable. The lowest formation energies are found for $\mathrm{P}_{\mathrm{Ga}}^{+2}$ in $p$-type and $\mathrm{V}_{\mathrm{Ga}}^{-3}$ in $n$-type GaP under P-rich conditions, and for $\mathrm{Ga}_{\mathrm{P}}^{-2}$ in $n$-type $\mathrm{GaP}$ under Ga-rich conditions. Finally, the finite size errors arising from the use of supercells with periodic boundary conditions are examined.
\end{abstract}

DOI: 10.1103/PhysRevB.72.195213

PACS number(s): 61.72.Ji, 71.55.Eq, 61.72.Bb, 71.15.Dx

\section{INTRODUCTION}

Due to its good optical properties, good performance and low cost, $\mathrm{GaP}$ is commonly found in semiconductor devices such as light emitting diodes (LEDs), long wavelength detectors and semiconductor lasers, as well as newer applications such as, for example, a host material for diluted magnetic semiconductors. The optical and electrical performance of the material is to a great extent controlled by the defects present, their type and, of course, density. When growing a semiconductor crystal it is impossible to create an ideal compound without grown-in native point defects. These will always be present to some extent and will thus affect the material properties. For example, deep level defects can act as nonradiative recombination centers which lower the performance of optical devices. Through a better understanding of the defects involved this could perhaps be counteracted and the efficiency improved. In the general case, this would yield higher quality materials and decrease the degradation and aging of devices.

Ideally one would like to get rid of all intrinsic defects in order to obtain a semiconductor as pure as possible before reintroducing certain types of defects under controlled conditions, i.e. doping the sample, to produce the desired material properties. The concentrations of these intentionally introduced impurities determine the performance of the semiconductor device but remaining native defects may act as compensators and therefore lower the concentration of charge carriers. In addition, a laser heterostructure will not remain very effective if the crucial dopants diffuse out of the active region, for instance, and the diffusion of dopants can often be either mediated or hindered by native point defects. Dopant diffusion rates can therefore be directly dependent on the concentrations of vacancies, antisites and selfinterstitials. In summary, the performance and reliability of the GaP optoelectronic devices is compromised by both the action of native defects such as nonradiative recombination centers and the out-diffusion of the electrically important dopants mediated by native defects.
Turning to previous work on defects in GaP, there have been numerous experiments relating to luminescence but the current knowledge of the electrical properties and structure of point defects is rather incomplete. However, various attempts have been made to identify specific defects. Experimental results are obtained which describe a defect with certain properties (spin, symmetry, or structure, for example). Then all possible candidates are lined up and, in the best case, ruled out one by one for different reasons, often by comparison to theory, until only one remains. It is therefore interesting to put this identification process into a larger perspective, calculating the relative abundances of the native point defects to see if this can provide an overall picture into which these individual identifications fit.

The experimental methods used have so far been electron spin resonance experiments (ESR) [or electron paramagnetic resonance (EPR)], ${ }^{1-8}$ deep-level transient spectroscopy (DLTS), ${ }^{9-11}$ optical detection of magnetic resonance (ODMR), ${ }^{12-15}$ and finally positron annihilation for the detection of vacancies. ${ }^{16,17}$ These studies almost always deal with one specific defect at a time so further discussion of them will be left to the relevant parts of Sec. III.

Most previous theoretical work relies on semiempirical and/or tight-binding calculations and does not include relaxations. ${ }^{18-21}$ Only three earlier studies include relaxations around the defects and only in a restricted sense; either the symmetry is fixed or only the nearest neighbors are allowed to relax. ${ }^{22-24}$ Furthermore, there is only one prior work, by Jansen and Sankey, ${ }^{25}$ actually dealing with the relative concentration of native defects in GaP through the calculation of formation energies. This is the only study which makes a comparison between different defects. In that work an $a b$ initio method with an $s p^{3}$ pseudo-atomic-orbital basis set was used with an 32 atom supercell. Relaxations were not taken into account. Their results will be compared to ours in Sec. IV when discussing the overall defect concentrations.

Apart from Jansen and Sankey, the previous defect studies in GaP only deal with one type of defect at a time and often 

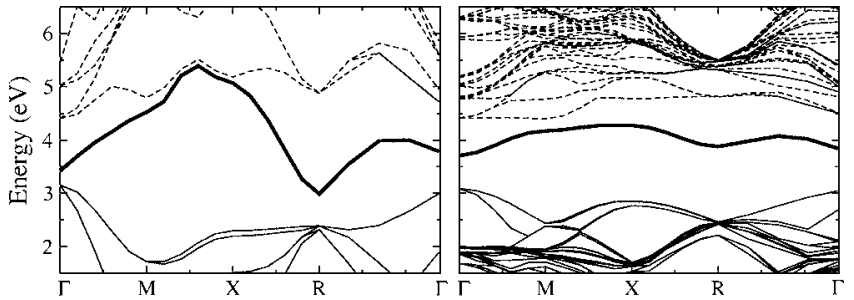

$\mathrm{k}(\mathrm{rad} / \mathrm{m})$

under very specific conditions. Here we attempt to present a more comprehensive picture of the native defects in GaP relating the different defects to each other, concentrating mainly on their density and stability for all their possible charge states. The dependence of the relative stability of a defect in a certain charge state on the overall doping of the material will be presented for all positions of the Fermi level ranging from strongly $p$-type to strongly $n$-type conditions. The knowledge of which defects occur the most at equilibrium under some specific conditions will shed new light on previous experimental identifications and controversies.

The paper is organized as follows. The theoretical methods are presented in Sec. II, where the computational method is described in detail and the expression used for the formation energy is derived along with the various conditions under which the work is performed. In Sec. III the results are presented for each of the native point defects. The electronic structure is related to the relaxed atomic configuration and bonding of the defects, for all the relevant charge states. The results are compared to experimental findings and previous theoretical work. Finally, in Sec. IV, the effects of the supercell approximation are considered, conclusions are drawn from the results and an overall picture of the native defects in $\mathrm{GaP}$ is presented.

\section{COMPUTATIONAL METHOD}

The calculations were performed using the VASP (Ref. 26) pseudopotential density functional theory ${ }^{27}$ (DFT) code in the local density approximation (LDA). Ultrasoft Vanderbilt pseudopotentials ${ }^{28}$ were used for the description of the $\mathrm{Ga}$ and $\mathrm{P}$ atoms ( $\mathrm{Ga} 3 d$ electrons were kept in the core) and plane waves were used as the basis set for the wave functions. The exchange-correlation part of the energy functional was based on the Ceperley-Alder potential ${ }^{29}$ in the parametrization by Perdew and Zunger. ${ }^{30}$ The calculations were performed in a cubic 216 atom supercell. Initial calculations used only the first special Chadi-Cohen ${ }^{31} k$-point, $\mathbf{k}$ $=\left(\frac{1}{4}, \frac{1}{4}, \frac{1}{4}\right)$, which gives better converged results than the gamma point. They were followed where necessary by more accurate calculations using a $2 \times 2 \times 2$ Monkhorst-Pack selection for both the ionic relaxations and the relaxed structures, and a $4 \times 4 \times 4$ Monkhorst-Pack ${ }^{32} k$-point selection for the ideal, unrelaxed structures. For the formation energy, defined below, this gave an accuracy of $0.05,0.01$, and $0.005 \mathrm{eV}$, respectively. A cutoff energy of $250 \mathrm{eV}$ was used for the plane waves which ensures an error of less than $0.01 \mathrm{eV}$ for the formation energies.
Ionic relaxations of the defect supercells were done using a Conjugated Gradient algorithm, starting from symmetrybroken geometries. No local geometries were maintained during the ionic relaxation and all atoms except the ones on the borders of the supercell were allowed to relax. The 216 atom supercell is sufficiently large that the condition of locking the border atoms does not impose serious restrictions on the relaxation of the host material surrounding the defect.

The calculations were performed with a non spinpolarized charge density and charged defects were calculated with a uniformly charged jellium background to ensure convergence of the total energy. There are various errors which arise from performing defect calculations using the supercell approximation. Because of the periodic boundary conditions there is an artificial electrostatic interaction between the defect charge distribution in neighboring supercells causing an additional electrostatic contribution to the energy. There is also a quantum-mechanical effect, since any overlap of the defect wave function between cells will cause a dispersion of the defect levels in the band gap. This is illustrated in Fig. 1 for the case of the phosphorous antisite $\left(\mathrm{P}_{\mathrm{Ga}}\right)$ in GaP. Therefore, small supercells will incorrectly describe the distribution of the defect states and it is required to use supercells large enough to remove this artificial interaction.

There is presently no good correction scheme for the supercell problems; the monopole term from the Makov-Payne correction is known to over correct the error, for example. In order to at least minimize the error, the largest computationally affordable supercell, the 216 atom supercell, has been used for the calculations presented here.

These (and other) finite size errors together with the LDA underestimation of the bandgap are the major sources of error in this work. The latter affects the energies of the defect levels making it very hard to quantitatively compare the positions of the charge transfer levels to experiment. The effects of finite size errors will be further discussed in Sec. IV.

The defect formation energy of a native defect is simply the reaction energy needed to create the defect from ideal material

$$
E_{\mathrm{def}}^{\mathrm{form}}(q)=E_{\mathrm{def}}^{\mathrm{tot}}(q)-E_{\mathrm{bulk}}^{\mathrm{tot}}-\Delta n_{\mathrm{Ga}} \mu_{\mathrm{Ga}}-\Delta n_{\mathrm{P}} \mu_{\mathrm{P}}+q\left(E_{F}+\varepsilon_{v}\right) .
$$

Here $E_{\mathrm{def}}^{\mathrm{tot}}(q)$ is the total energy of the supercell containing the defect and $E_{\text {bulk }}^{\text {tot }}$ is the total energy of the pure bulk crystal, calculated in the same supercell. $\Delta n_{i}$ is the number of atoms of species $i$ added from a particle reservoir of chemical potential $\mu_{i}$ in order to create the defect. $q$ is the charge state of the defect and hence equal to the number of electrons 
taken away from the supercell in order to create the charged defect and $E_{F}$ is the Fermi level, i.e., the electron chemical potential, from which electrons are taken to charge the defect. By convention $E_{F}$ is set to zero at the valence band maximum $\varepsilon_{v}$, which is obtained from a band-structure calculation at the $\Gamma$ point in bulk GaP, using the charge density from a $k$-point converged bulk calculation. ${ }^{33}$

The insertion of a defect into the supercell causes the electrostatic potential to shift relative to that of the bulk supercell. As a consequence, the zero energy levels no longer coincide exactly, so that formation energies calculated using this value of $\varepsilon_{v}$ contain an additional error. This can be corrected for by aligning the potential in the defect supercell in a bulklike region far from the defect with that of a bulk supercell at the same location. ${ }^{33}$ However, in this work it is found that for the 216 atom supercell this potential shift is less than $0.04 \mathrm{eV}$ and therefore any direct correction is neglected. Furthermore, this error is proportional to the cell volume, so it will in fact be corrected when finite size errors are examined in the last section.

The chemical potentials are parameters which depend upon the conditions under which the material has been grown or prepared. However, there are restrictions that limit the possible values for these parameters and determine the possible range of the Ga-P balance and stoichiometry. First, the chemical potentials must be lower than those of the corresponding bulk materials, that is, $\mu_{\mathrm{Ga}} \leqslant \mu_{\mathrm{Ga}}^{\mathrm{bulk}}$ and $\mu_{\mathrm{P}}$ $\leqslant \mu_{\mathrm{P}}^{\text {bulk }}$, otherwise the pure bulk materials will be more stable and form instead. The upper limits are referred to as the gallium-rich and phosphorus-rich limits. Secondly, for a system in equilibrium at constant temperature and pressure, the following relation for the chemical potentials must be fulfilled,

$$
\mu_{\mathrm{GaP}}=\mu_{\mathrm{Ga}}+\mu_{\mathrm{P}}
$$

where $\mu_{\mathrm{GaP}}$ is the chemical potential of bulk GaP. This leaves only one free parameter, here chosen to be $\mu_{\mathrm{Ga}}$, which is restricted to the interval

$$
\mu_{\mathrm{GaP}}-\mu_{\mathrm{P}}^{\text {bulk }} \leqslant \mu_{\mathrm{Ga}} \leqslant \mu_{\mathrm{Ga}}^{\text {bulk }} .
$$

The upper limit is the gallium-rich case and the lower limit the phosphorus-rich case.

The bulk chemical potentials are taken from fully converged calculations for the respective materials. The numerical values used are $\mu_{\mathrm{Ga}}^{\text {bulk }}=-3.61 \mathrm{eV}, \mu_{\mathrm{P}}^{\text {bulk }}=-6.03 \mathrm{eV}$ (black phosphorus), and $\mu_{\mathrm{GaP}}=-10.53 \mathrm{eV}$.

In addition, unless the defect is neutral, the formation energy will also be dependent on the Fermi energy, which means that the formation energy is a function of both the stoichiometry and the doping of the $\mathrm{GaP}$ semiconductor, $E^{\text {form }}=E^{\text {form }}\left(\mu_{\mathrm{Ga}}, E_{F}\right)$.

The concentration of a defect can now be calculated using the formation energy. Minimizing the Gibbs free energy for the system under the assumptions that the defect concentration is small and independent of other defects and that the pressure and volume are constant gives the equilibrium concentration

$$
[C]=N e^{-E^{\mathrm{form}} / k_{B} T} e^{S_{f} / k_{B}} .
$$

Here $k_{B}$ is the Boltzmann constant, $T$ the temperature in $\mathrm{K}, N$ is the concentration of possible lattice sites or interstitial sites for the defect, and $S_{f}$ is the formation entropy (including all entropy contributions except for the configurational part arising from the selection of a defect lattice site). This entropy term is dominated by vibrational contributions and is in the range of $0-10 k_{B}$, with typical values of $5-6 k_{B}$ for point defects in Si (Refs. 34 and 35) (corresponding to a lowering of the formation energy by $0.13-0.16 \mathrm{eV})$. This can therefore give an additional contribution and raise the concentration by as much as four orders of magnitude.

The exponential dependence of this equation means that a very small change in the formation energy can change the defect concentration by several orders of magnitude. The concentrations are therefore very sensitive to errors in the formation energy and it is only reasonable to estimate concentrations in orders of magnitude. The number of possible lattice sites will, of course, also alter the defect concentration by (at most) one order of magnitude but in this study this number is equal for all the defects considered: $2.5 \times 10^{22} \mathrm{~cm}^{-3}$. Because of this the relative abundances of the native defects can be seen directly from a comparison of the formation energies.

Note that the defects in this study are considered to be incorporated into the crystal during growth and hence there will be no need to strictly maintain the Ga to $\mathrm{P}$ balance. This means that defect concentrations determined via Eq. (4) will only be dependent on the formation energy of the defect in question.

Using the method described above the lattice constant of the zinc-blend GaP structure is calculated to be $5.39 \AA$, with an error of $1 \%$ relative to the experimental value of $5.45 \AA$. $\mathrm{GaP}$ has an indirect bandgap with a conduction band minimum which is here calculated to be at $\mathbf{k}=(0.87,0,0)$, giving a DFT-LDA bandgap of $1.42 \mathrm{eV}$. This is to be compared to the experimental conduction band minimum at $\mathbf{k}$ $=(0.95,0,0)$ and bandgap of $2.35 \mathrm{eV} \cdot{ }^{36}$ The results presented in this work (Figs. 3-6,8) will be given with reference to the theoretical bandgap, rather than being plotted all the way up to the experimental band edge (the extended zone scheme). The validity of this is the subject of a forthcoming investigation. ${ }^{37}$

\section{RESULTS}

The native defects investigated in this study are the antisites on the $\mathrm{Ga}$ and $\mathrm{P}$ sublattices $\left(\mathrm{P}_{\mathrm{Ga}}, \mathrm{Ga}_{\mathrm{P}}\right)$, the $\mathrm{Ga}$ and $\mathrm{P}$ interstitials located at either the $\mathrm{P}$-surrounded or $\mathrm{Ga}$ surrounded tetragonal sites $\left(\mathrm{P}_{i: \mathrm{P}}, \mathrm{P}_{i: \mathrm{Ga}}, \mathrm{Ga}_{i: \mathrm{P}}, \mathrm{Ga}_{i: \mathrm{Ga}}\right)$ and finally the $\mathrm{Ga}$ and $\mathrm{P}$ vacancies $\left(\mathrm{V}_{\mathrm{Ga}}, \mathrm{V}_{\mathrm{P}}\right)$. The calculations were performed for all relevant charge states of each defect. For the purpose of making the discussion of the different charge states of the defects clearer, the schematics of the LDA Kohn-Sham defect levels in the band gap for the neutral charge states are shown in Fig. 2. The charge states that are stable in the bandgap have neither electrons in the conduction band nor holes in the valence band. It is correct to 


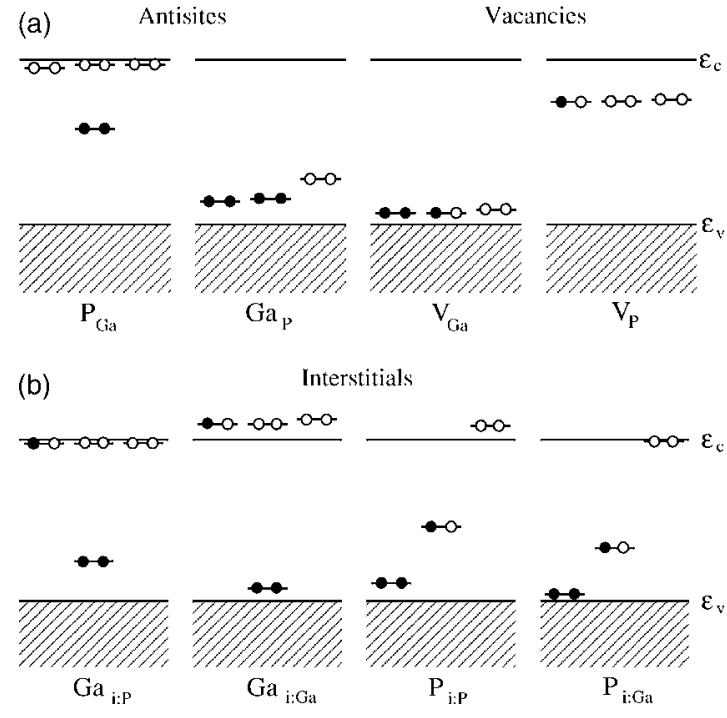

FIG. 2. Schematic for the defect levels at $\mathbf{k}=\left(\frac{1}{4}, \frac{1}{4}, \frac{1}{4}\right)$ for the neutral defects.

talk about a certain charge state of a defect when any electrons or holes introduced in forming it occupy defect states within the bandgap and are therefore localized on or around the defect site. $\left(\mathrm{Ga}_{i: \mathrm{Ga}}^{0}\right.$ in Fig. 2 is never stable within the bandgap, as will be shown later).

Preliminary calculations were performed using the 64 atom simple cubic supercell in order to determine the stable charge states for each of the defects directly instead of relying on the possible charge states suggested by the defect levels of the neutral defect. However, the 64 atom supercell imposes such a large dispersion of the defect levels that it is found to be necessary to use the 216 atom simple cubic cell to get reliable results. (For the $\mathrm{P}$ antisite the dispersion of the deep gap level in the eight atom supercell, measured as the difference between the maximum and minimum energies, is $2.4 \mathrm{eV}$ and remains as large as $0.6 \mathrm{eV}$ in the 64 atom cell. However, in the 216 cell the dispersion is less than $0.1 \mathrm{eV}$, as shown in Fig. 1). This dispersion can, of course, lead to further errors in the calculated formation energies. It has been suggested ${ }^{7}$ that these additional errors arise when the formation energy is calculated using special $k$-points (as we do) rather than just the Gamma point. It is then suggested that they should be corrected by adding a factor $q \cdot\left(\epsilon_{D}^{k}-\epsilon_{D}^{\Gamma}\right)$, where $\epsilon_{D}^{k}$ and $\epsilon_{D}^{\Gamma}$ are the values of the defect level in the gap calculated as the average over the special $k$-points and at the $\Gamma$ point, respectively, both for the neutral charge state of the defect. This "correction" makes the implicit assumption that the defect level in smaller supercells is essentially correct at the $\Gamma$ point and incorrect at the special $k$-points. Figure 1 shows very clearly that, at least for the example of $\mathrm{P}_{\mathrm{Ga}}$, this is not at all the case. The mean value of the very dispersed levels in the 8 and 64 atom supercell is rather close to the value of the fairly flat defect level in the 216 atom cell, which we can take as a reasonable approximation to the infinite limit in this case. However, the $\Gamma$ point values of the defect level in the smaller cells are much less accurate. As a result the "correction" suggested will for this example actually make matters considerably worse, so such corrections

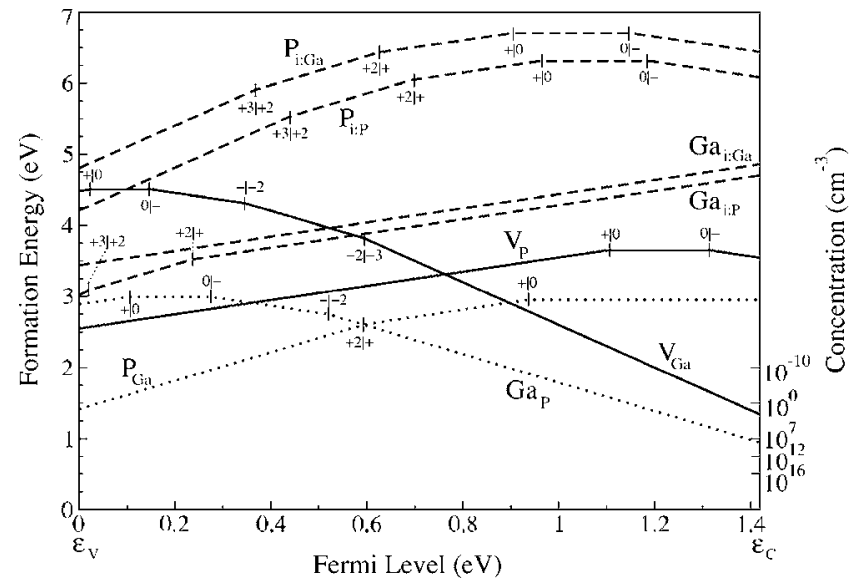

FIG. 3. Formation energies as a function of the Fermi-level for all unrelaxed native defects under stoichiometric conditions $\left(\mu_{\mathrm{Ga}}\right.$ $=-4.06 \mathrm{eV}$ ). Values are only shown for the most stable charge state at any Fermi-level, the charge state given by the slope of the line. Charge transfer levels are labeled next to the vertical lines indicating their positions. The antisite defects are shown as dotted lines, vacancies as full lines and interstitials as broken lines. The alternative $y$ axis on the right gives the defect concentration in orders of magnitude at a temperature of $300 \mathrm{~K}$.

will be ignored in the current work. The effects of dispersion will instead be dealt with along with other finite size effects in Sec. IV.

When calculating formation energies, the defect is considered to be an isolated defect surrounded by a macroscopic host crystal under doping conditions which determine the Fermi-level. In this way the Fermi level is fixed at a certain value in the bandgap and the calculated formation energy is the energy required to create a point defect at that particular Fermi level. These formation energies are plotted in Fig. 3 for the unrelaxed defects and in Figs. 4-6 for the fully relaxed defects, all as a function of the Fermi level across the bandgap. Here the relaxation energies have been calculated using the $\mathbf{k}=\left(\frac{1}{4}, \frac{1}{4}, \frac{1}{4}\right)$ point (it has been shown ${ }^{38}$ that the re-

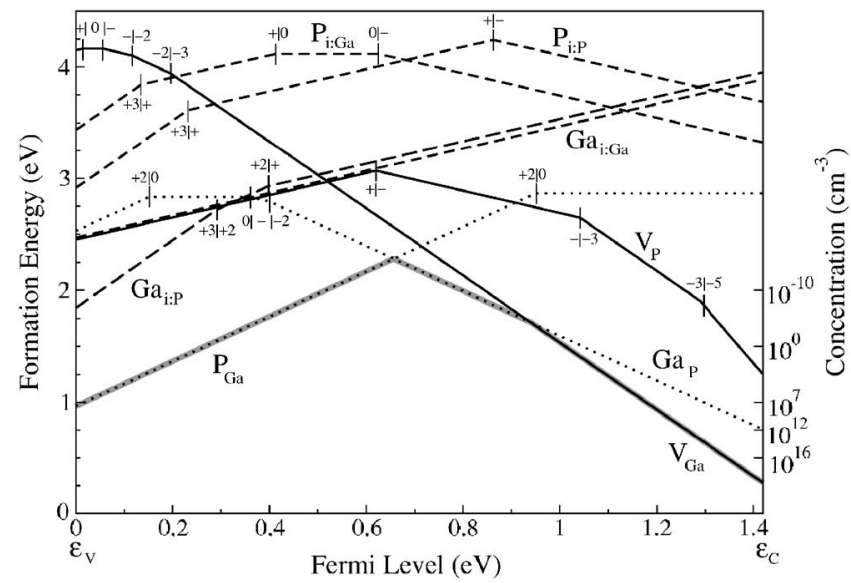

FIG. 4. As Fig. 3, but for all the fully relaxed native defects under stoichiometric conditions $\left(\mu_{\mathrm{Ga}}=-4.06 \mathrm{eV}\right)$. The most stable type of defect for each value of the Fermi-level is shown by the thick grey line. 


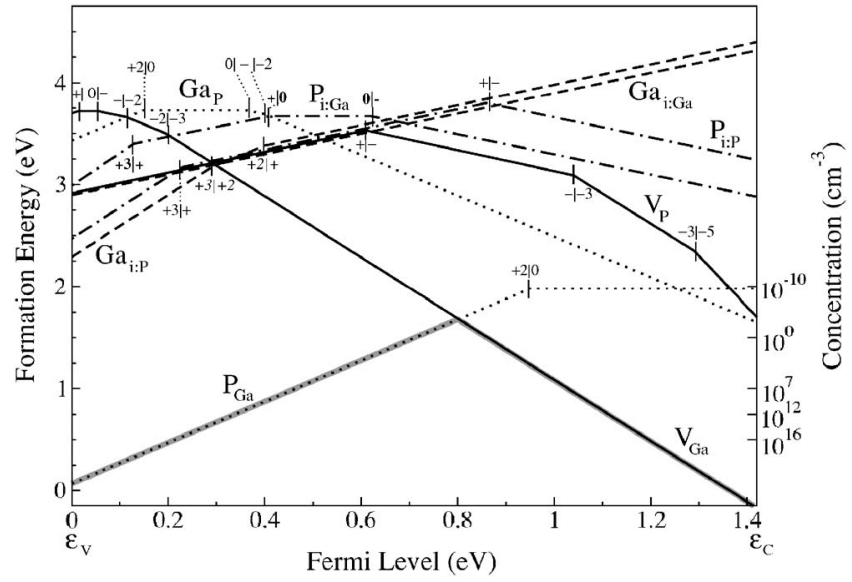

FIG. 5. As Fig. 4, but under P-rich conditions $\left(\mu_{\mathrm{Ga}}=-4.50 \mathrm{eV}\right)$.

laxation energy converges faster with $k$-point density than the formation energy itself). From Eq. (1) it is seen that the formation energy depends linearly on the Fermi level so that the slope of the curve corresponds to the charge state of the defect. In the figures, only the defect's most stable charge state is shown for each value of the Fermi level. (Higher and lower charge states than the ones shown in the figures have also been calculated to make sure that the charge states shown for $E_{F}=\varepsilon_{v}$ and $E_{F}=\varepsilon_{c}$ are indeed the most stable.) The special values of the Fermi level for which the most stable charge state of a particular defect changes, i.e., the charge transfer levels, are indicated in the figures by vertical lines labeled with the relevant charge states.

For the defects with the lowest formation energies, which are expected to occur in the highest concentrations, the relaxations were refined using a $2 \times 2 \times 2$ Monkhorst-Pack $k$-point grid and a higher convergence criterion for relaxation. This was done for all charge states of the antisite defects $\left(\mathrm{P}_{\mathrm{Ga}}\right.$ and $\left.\mathrm{Ga}_{\mathrm{P}}\right)$ and the $\mathrm{P}$ vacancy $\left(\mathrm{V}_{\mathrm{P}}\right)$ as well as for the gallium vacancy in the -3 charge state $\left(\mathrm{V}_{\mathrm{Ga}}^{-3}\right)$. It results in more completely relaxed structures of increased symmetry, which will be used in the structural discussions. However, it

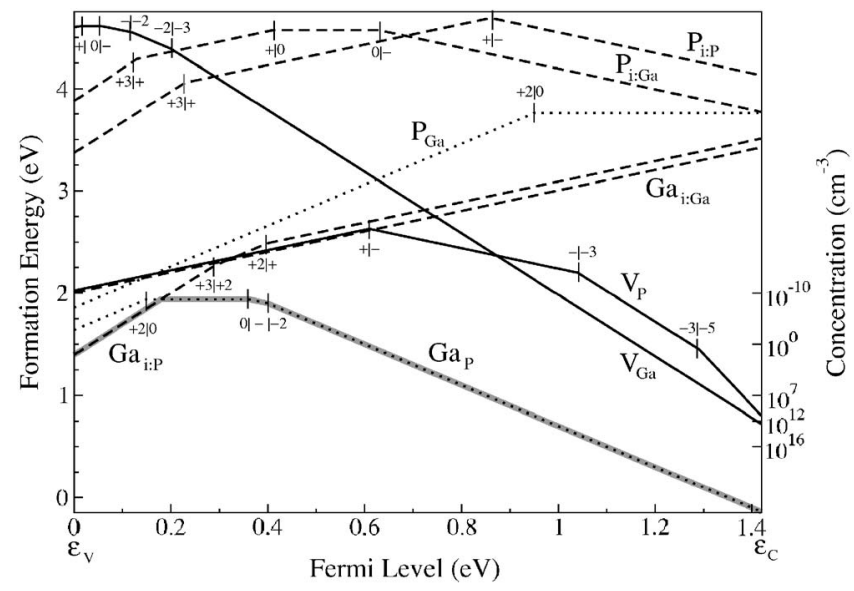

FIG. 6. As Fig. 4, but under Ga-rich conditions $\left(\mu_{\mathrm{Ga}}=-3.61 \mathrm{eV}\right)$. changes the formation energies by less than $0.05 \mathrm{eV}$ so Figs. 4-6 only display the results obtained using $\mathbf{k}=\left(\frac{1}{4}, \frac{1}{4}, \frac{1}{4}\right)$ for the sake of consistency.

\section{A. Phosphorus vacancy}

The ideal $\mathrm{P}$ vacancy has a localized $A_{1}$ defect state inside the valence band and a threefold degenerate $T_{2}$ state in the upper part of the bandgap, close to the conduction band. In the neutral state there is one electron occupying this $T_{2}$ level (shown in Fig. 2 for the relaxed vacancy). Therefore $V_{P}$ may be expected to take any charge state from +1 to -5 depending on the position of $E_{F}$. The P vacancy undergoes considerable relaxation and different charge states have very different structures. Moving from the positive to the more negative charge states; $\mathrm{V}_{\mathrm{P}}^{+1}$ is the most energetically favorable charge state for values of the Fermi level in the lower part of the bandgap (Fig. 4). The tetragonal configuration is maintained after relaxation, as expected since all defect levels in the bandgap are empty for this charge state. The Ga-Ga distance between the four nearest-neighbors is lowered from $3.81 \AA$, in the unrelaxed case, to $3.69 \AA$. This corresponds to a decrease in the vacancy volume of $9.4 \%$ (see Table I).

For the neutral vacancy $\mathrm{V}_{\mathrm{P}}^{0}$, the tetrahedral symmetry is broken and the symmetry is reduced to $D_{2 d}$. Dimers are formed between two pairs of Ga nearest neighbors, although only one electron is distributed over the two bonds since the first defect level is only partially occupied. These dimers correspond to two opposite sides of the nearest-neighbor tetrahedron being equal to $3.16 \AA$ while the other four sides have a length of $3.49 \AA$. The vacancy volume is now $4.46 \AA$ corresponding to a reduction of $31.6 \%$.

Completely filling one of the (now nondegenerate) defect levels originating from the $T_{2}$ level and leaving the other two empty forms the $\mathrm{V}_{\mathrm{P}}^{-1}$ state. As with the neutral vacancy, the symmetry is reduced to $D_{2 d}$ with dimers forming between two pairs of Ga nearest neighbors. These are now fully occupied, resulting in a tetragonal structure that has two opposite sides equal to $2.74 \AA$ and the remaining four sides equal to $3.42 \AA$. This reduces the volume further, giving an inward relaxation of $46.0 \%$ from the ideal volume. This distinct change in bonding structure lowers the formation energy from 4.97 to $3.69 \mathrm{eV}$ at $E_{F}=0$ and causes a reverse ordering of the $(+\mid 0)$ and $(0 \mid-)$ charge transfer levels, resulting in a so called "negative-U" (+|-) transfer level (Fig. 4). This reverse ordering of the charge transfer levels is a relaxation effect. If the local structure is kept fixed at the full $T_{d}$ symmetry and two electrons are added to the system, the second electron will always raise the energy more than the first one because of electron-electron repulsion and this will ensure the normal ordering. In this case however, the additional relaxation energy of the -1 charge state makes it energetically favorable to fill the lowest defect level by two electrons at the same time and therefore the neutral charge state is never stable.

Inserting one more electron, partially occupying the second defect level, forms the $\mathrm{V}_{\mathrm{P}}^{-2}$ charge state. This does not result in any qualitatively new bonding structure and the largest difference compared to the singly negative charge state is the continued decrease of the vacancy volume to 
TABLE I. Table over relaxation effects: Columns three and four give the formation energies for the unrelaxed and relaxed defects, respectively. The values are calculated for $E_{F}=\varepsilon_{v}$. In column five the defect volume is given as the tetrahedral volume formed by the four nearest neighbors (not shown for hexagonal and bond centered interstitials). Column six gives the percentage change in this volume upon relaxation from the ideal structure. Negative values are defined as inward relaxations. Columns seven and eight indicate the symmetry of the relaxed defect and the change in bond lengths as a percentage of the average bond length going from $T_{d}$ to the relaxed structure. The last column shows the degree of distortion from the relaxed symmetry, quantified by expressing the maximal deviation in nearest neighbor distances as a percentage of their average (this is essentially a measure of the remaining errors in the relaxed structure).

\begin{tabular}{|c|c|c|c|c|c|c|c|c|}
\hline Defect & $\mathrm{q}$ & $\begin{array}{l}E_{\text {static }}^{\text {form }} \\
{[\mathrm{eV}]}\end{array}$ & $\begin{array}{c}E_{\text {rolaxed }}^{\text {form }} \\
{[\mathrm{eV}]}\end{array}$ & $\begin{array}{c}\text { Volume } \\
{\left[\AA^{3}\right]}\end{array}$ & $\begin{array}{l}\text { Vol. Relax. } \\
\quad[\%]\end{array}$ & Sym. & $\begin{array}{c}\text { Change from } T_{d} \\
{[\%]}\end{array}$ & $\begin{array}{l}\text { Dist. } \\
{[\%]}\end{array}$ \\
\hline \multirow[t]{7}{*}{$\mathrm{V}_{\mathrm{P}}$} & +1 & 2.55 & 2.45 & 5.91 & -9.4 & $T_{d}$ & 0.00 & 0.02 \\
\hline & 0 & 3.65 & 3.33 & 4.46 & -31.6 & $D_{2 d}$ & 9.68 & 0.04 \\
\hline & -1 & 4.97 & 3.69 & 3.52 & -46.0 & $D_{2 d}$ & 21.4 & 0.01 \\
\hline & -2 & 6.42 & 4.89 & 3.18 & -51.2 & $D_{2 d}$ & 16.2 & 5.76 \\
\hline & -3 & 7.89 & 5.77 & 2.70 & -58.6 & $D_{2 d}$ & 15.3 & 0.09 \\
\hline & -4 & 9.41 & 7.18 & 2.57 & -60.6 & $D_{2 d}$ & 9.64 & 4.62 \\
\hline & -5 & 10.97 & 8.35 & 2.35 & -64.0 & $T_{d}$ & 0.00 & 0.02 \\
\hline \multirow[t]{5}{*}{$\mathrm{V}_{\mathrm{Ga}}$} & +1 & 4.48 & 4.15 & 4.55 & -30.2 & $C_{3 v}$ & 1.36 & 0.01 \\
\hline & 0 & 4.51 & 4.17 & 4.61 & -29.3 & $C_{3 v}$ & 1.23 & 0.64 \\
\hline & -1 & 4.66 & 4.22 & 4.57 & -29.9 & $C_{3 v}$ & 1.22 & 0.72 \\
\hline & -2 & 5.00 & 4.34 & 4.50 & -31.0 & $C_{3 v}$ & 1.28 & 0.81 \\
\hline & -3 & 5.60 & 4.53 & 4.35 & -33.3 & $T_{d}$ & 0.00 & 0.00 \\
\hline \multirow[t]{3}{*}{$\mathrm{Ga}_{i: \mathrm{P}}$} & +3 & 3.02 & 1.84 & 6.76 & +3.7 & $T_{d}$ & 0.00 & 0.10 \\
\hline & +2 & 3.05 & 2.14 & 6.98 & +7.1 & $T_{d}$ & 0.00 & 0.23 \\
\hline & +1 & 3.28 & 2.53 & 7.27 & +11.5 & $T_{d}$ & 0.00 & 0.30 \\
\hline $\mathrm{Ga}_{i: \mathrm{Ga}}$ & +1 & 3.44 & 2.45 & 8.45 & +29.6 & $T_{d}$ & 0.00 & 0.09 \\
\hline \multirow[t]{5}{*}{$\mathrm{P}_{i: \mathrm{P}}$} & +3 & 4.22 & 2.916 & 6.24 & -4.3 & $T_{d}$ & 0.00 & 0.21 \\
\hline & +2 & 4.66 & 3.45 & - & - & $C_{3 v}$ & - & - \\
\hline & +1 & 5.35 & 3.38 & - & - & $C_{3 v}$ & - & - \\
\hline & 0 & 6.31 & 4.29 & - & - & $C_{1 h}$ & - & - \\
\hline & -1 & 7.50 & 5.11 & - & - & $C_{1 h}$ & - & - \\
\hline \multirow[t]{5}{*}{$\mathrm{P}_{i: \mathrm{Ga}}$} & +3 & 4.80 & 3.44 & 8.98 & +37.7 & $T_{d}$ & 0.00 & 0.32 \\
\hline & +2 & 5.18 & 4.22 & - & - & $C_{3 v}$ & - & - \\
\hline & +1 & 5.80 & 3.71 & - & - & $C_{1}$ & - & - \\
\hline & 0 & 6.71 & 4.12 & - & - & $C_{1}$ & - & - \\
\hline & -1 & 7.86 & 4.74 & - & - & $C_{1}$ & - & - \\
\hline \multirow[t]{3}{*}{$\mathrm{P}_{\mathrm{Ga}}$} & +2 & 1.41 & 0.97 & 5.70 & -12.6 & $T_{d}$ & 0.00 & 0.01 \\
\hline & +1 & 2.01 & 1.94 & 6.31 & -3.2 & $T_{d}$ & 0.00 & 1.35 \\
\hline & 0 & 2.95 & 2.87 & 7.00 & +7.4 & $T_{d}$ & 0.00 & 0.06 \\
\hline \multirow[t]{5}{*}{$\mathrm{Ga}_{\mathrm{P}}$} & +2 & 2.89 & 2.53 & 7.57 & +16.1 & $T_{d}$ & 0.00 & 0.02 \\
\hline & +1 & 2.89 & 2.69 & 7.27 & +11.5 & $C_{2 v}$ & 4.10 & 0.35 \\
\hline & 0 & 2.99 & 2.83 & 6.87 & +5.4 & $D_{2 d}$ & 6.16 & 0.32 \\
\hline & -1 & 3.27 & 3.19 & 6.57 & +0.8 & $C_{2 v}$ & 3.72 & 0.59 \\
\hline & -2 & 3.79 & 3.59 & 6.24 & -4.3 & $T_{d}$ & 0.00 & 0.00 \\
\hline
\end{tabular}


$3.18 \AA^{3}$. The last electron, occupying the highest defect level, therefore causes an additional electron-electron repulsion which makes this charge state less favorable. In fact $\mathrm{V}_{\mathrm{P}}^{-2}$ is never stable, since filling up the second defect level, to produce the $\mathrm{V}_{\mathrm{P}}^{-3}$ state, again drastically changes the bonding structure allowing strong gains in relaxation energy leading to a $(-\mid-3)$ negative- $U$ charge transfer level. For $V_{P}^{-3}$ the bonding is strengthened along two more Ga-Ga distances giving four short distances of $2.76 \AA$ and two long distances, opposite to each other, of $3.21 \AA$. The symmetry group therefore is still $D_{2 d}$ although the structure is different. The stronger bonding results in an even smaller vacancy volume, $2.70 \AA^{3}$.

In the $\mathrm{V}_{\mathrm{P}}^{-4}$ state the third and highest defect level, which is located just below the conduction band, is partially occupied. As with the -2 charge state the structure is not much altered from the preceding one (the two long distances are now equal to $3.00 \AA$ and the four short, in average, $2.73 \AA$ ). The volume is in fact only reduced from $2.70 \AA^{3}$ to $2.57 \AA^{3}$ and as before the unpaired electron leads to a relatively high formation energy.

In the $\mathrm{V}_{\mathrm{P}}^{-5}$ state the last defect level is fully occupied and moves down to the same energy as the other two levels in the bandgap. The structure changes back to a high symmetry configuration with the full $T_{d}$ symmetry and a relaxation energy of $2.62 \mathrm{eV}$ resulting yet again in a reverse ordering and a $(-3 \mid-5)$ charge transfer level (Fig. 4). There is a systematic reduction of the $\mathrm{P}$ vacancy volume when increasing the number of localized electrons, the overall smallest volume being $2.35 \AA$ for $\mathrm{V}_{\mathrm{P}}^{-5}, 364.0 \%$ smaller than the ideal vacancy volume. This great reduction arises because with five electrons added, equal to the number of $\mathrm{P}$ valence electrons removed when creating the vacancy, the dangling bonds on each of the four surrounding $\mathrm{Ga}$ atoms are completely filled. The filling of these bonds gives $\mathrm{Ga}-\mathrm{Ga}$ nearest-neighbor distances of $2.71 \AA$. In Table $\mathrm{I}$ it is seen that for the $\mathrm{V}_{\mathrm{P}}^{-2}$ and $\mathrm{V}_{\mathrm{P}}^{-4}$ states, which are never the most stable, the relaxation convergence is poor indicating that maybe they are not even metastable.

From Fig. 4 it is seen that in $p$-type $\mathrm{GaP} \mathrm{V}_{\mathrm{P}}^{+1}$ will be the most stable charge state, in semi-insulating $\mathrm{V}_{\mathrm{P}}^{-1}$ is the most stable, and in $n$-type the $\mathrm{V}_{\mathrm{P}}^{-3}$ state has the lowest formation energy, except in very strongly $n$-type material in which $\mathrm{V}_{\mathrm{P}}^{-5}$ has an even lower energy. According to the formation energies in Fig. 4, the highest concentration of $V_{P}$ occurs in strongly $n$-type $\mathrm{GaP}$ in the form of the $\mathrm{V}_{\mathrm{P}}^{-5}$ state.

Regarding the stoichiometry, $\mathrm{V}_{\mathrm{P}}$ is expected to be more favorable in $\mathrm{GaP}$ grown under $\mathrm{Ga}$-rich conditions than under P-rich conditions, as confirmed by a comparison of Figs. 5 and 6. The difference in defect formation energy is $0.9 \mathrm{eV}$ which corresponds to a considerable difference in $V_{P}$ concentration between different growth conditions.

It is interesting to compare the present calculations to the cluster calculations of Khoo and Ong since, while less accurate in most other respects, the cluster calculations do not suffer from any dispersion of the defect levels. ${ }^{22}$ They used an empirical self-consistent molecular-orbital method with complete neglect of differential overlap (CNDO) for clusters consisting of 72 atoms. Relaxations were included for the four $\mathrm{Ga}$ atoms surrounding the vacancy and the parameters were adjusted to give good agreement between experimental results and those of pure bulk GaP calculations. They further considered only the $\mathrm{V}_{\mathrm{P}}^{+1}, \mathrm{~V}_{\mathrm{P}}^{0}$, and $\mathrm{V}_{\mathrm{P}}^{-1}$ charge states.

For $\mathrm{V}_{\mathrm{P}}^{+1}$ the two calculations agree that the full $T_{d}$ symmetry is maintained. However, the cluster calculation produced an outward relaxation, moving the Ga nearest neighbors away from the vacancy site by $4.0 \%$ of the ideal Ga-P bond length whereas in this work the change is $0.8 \%$ inwards. Khoo and Ong also found outward relaxations for $V_{\mathrm{P}}^{0}$ and $\mathrm{V}_{\mathrm{P}}^{-1}$ in disagreement with our results and for these there is no agreement on the symmetry of the relaxed structures either. These major disagreements regarding the relaxation can be explained by the very restricted relaxation in the cluster calculation, where only the four $\mathrm{Ga}$ atoms surrounding the vacancy site were allowed to relax, and the lower accuracy of their computational method. In this paper we find that $\mathrm{V}_{\mathrm{P}}^{+1}$ undergoes an inward relaxation, reducing the vacancy volume, although the distances between the $\mathrm{Ga}$ atoms and their three $\mathrm{P}$ neighbors (the next nearest neighbors of the vacancy) are still very close to the ideal Ga-P distance of $2.33 \AA$. This means that the relaxation concerns many shells around the vacancy and it is therefore insufficient to relax only the four nearest $\mathrm{Ga}$ atoms.

This conclusion is supported by a more recent study by Schwarz et al. who, using pseudopotential DFT calculations in a 64 atom bulk supercell, also found an inward relaxation for $\mathrm{V}_{\mathrm{P}}^{0}$ in $\mathrm{GaP}^{23}$ The symmetries of the relaxed structures disagree though; they found that the full $T_{d}$ symmetry was maintained while we find that the symmetry broken structure described above has a formation energy $0.1 \mathrm{eV}$ lower than that of the tetragonal structure.

Despite the structural differences between the computational methods Khoo also found a reverse ordering of the $(0 \mid-)$ and $(+\mid 0)$ charge transfer levels in accordance with the $(+\mid-)$ level in Fig. 4 found here. The vacancies in $\mathrm{GaP}$ have been studied by Krause-Rehberg et al. ${ }^{17}$ using positron annihilation. They varied the Fermi level through the upper half of the bandgap by changing the temperature and introducing compensating deep level defects. The position of the Fermi level was then measured using the Hall effect. Different charge states have different trapping behavior and through this the transfer levels can be identified as the values of the Fermi level for which the trapping rate abruptly changes. No positron trapping was observed in $p$-type or semi-insulating material but there was a large trapping found in $n$-type material. This is consistent with the results presented here since $\mathrm{V}_{\mathrm{P}}^{+1}$ is most stable in $p$-type $\mathrm{GaP}$ and positive vacancies repel positrons and are therefore not detectable by this technique. In $n$-type material, on the other hand, a vacancy concentration on the order of $10^{16} \mathrm{~cm}^{-3}$ is observed and ascribed by Krause-Rehberg et al. to negatively charged $\mathrm{P}$ vacancies, which according to our results would be $\mathrm{V}_{\mathrm{P}}^{-3}$ and $\mathrm{V}_{\mathrm{P}}^{-5}$. (See the gallium vacancy section and Discussions and Conclusions for further discussion of these experimental findings, however). From a parameter model fit to the positron annihilation data they obtained a charge transfer level at $250 \mathrm{meV}$ below the conduction band. Based on comparisons to an early tight-binding calculation for the ideal vacancy they assumed this to be the $\mathrm{V}_{\mathrm{P}}(0 \mid-)$ level. ${ }^{39}$ Looking at Fig. 4 it is instead more likely that this experimental result is due 
to the $(-3 \mid-5)$ charge transfer level, although exact comparisons of the charge transfer levels cannot be made because of the LDA bandgap errors. Never-the-less, no other charge transfer levels are observed within $400 \mathrm{meV}$ of the conduction band and according to Fig. 4 the $(-3 \mid-5)$ level is the only charge transfer level in the upper part of the bandgap originating from vacancies.

\section{B. Gallium vacancy}

Similar to the $\mathrm{P}$ vacancy, the ideal Ga vacancy is expected to have an $A_{1}$ defect level inside the valence band and a triply degenerate $T_{2}$ state in the bandgap, which is occupied by three electrons in the neutral charge state. For the unrelaxed $\mathrm{V}_{\mathrm{Ga}}$ the distance between the four $\mathrm{P}$ atoms forming the tetrahedron around it is $3.81 \AA$. The main effect of the relaxation is that this tetrahedron undergoes a large inward relaxation, resulting in a P-P distance of around $3.4 \AA$, with only minor variations between different charge states. The triply degenerate $T_{2}$ level is, for all except the -3 charge state, further split into a singlet state and a doubly degenerate level, as shown in Fig. 2, since one $\mathrm{P}$ atom is moved about $0.05 \AA$ closer to the vacancy site than the other three, reducing the symmetry to $C_{3 v}$. Nevertheless the deviation from the $T_{d}$ symmetry is never more than $1.8 \%$ of the neighborneighbor distance for any charge state.

The inward relaxation is in agreement with the results of Schwarz et al. for the neutral defect. They found that the four P nearest-neighbor atoms all move $3.7 \%$ of the ideal bonding distance towards the vacancy site. The corresponding number here is $4.4 \%$. The slightly larger relaxation can be explained by the larger supercell used and that the relaxation is less restricted here.

From the formation energy plots in Fig. 4 it is clear that the only relevant charge state is the triply negative one. Although the charge states $+1 \rightarrow-2$ should occur in $p$-type material their high formation energies and hence vanishingly small relative concentrations make them irrelevant. Therefore only the $\mathrm{V}_{\mathrm{Ga}}^{-3}$ structure will be discussed in further detail. Since atomic Ga has three valence electrons there are three electrons missing from the neutral vacancy. In $\mathrm{V}_{\mathrm{Ga}}^{-3}$ the dangling bond on each of the four surrounding $\mathrm{P}$ atoms is completely occupied, filling all the defect levels in the bandgap. The $\mathrm{P}$ atoms can therefore maintain their $s p^{3}$ bonding structure and no symmetry breaking is then expected. This is indeed the case, and the structure relaxes back to the $T_{d}$ symmetry when distorted (see Table I). The P-P distances of the tetrahedron surrounding the vacancy are reduced from 3.81 to $3.33 \AA$ : a $33.3 \%$ decrease in the vacancy volume. In spite of this considerable inward relaxation the nearestneighbor to second nearest-neighbor distances are 0.02-0.03 $\AA$ smaller than the ideal Ga-P distance in defect free material. The large inward relaxation instead comes from Ga-P distance elongations in the second and third shells and (decreasingly) further out. In this way $\mathrm{V}_{\mathrm{Ga}}^{-3}$ causes a widespread disturbance in the surrounding crystal structure.

Considering the stoichiometry, $\mathrm{V}_{\mathrm{Ga}}^{-3}$ is the most common of all the native defects considered in this study for $n$-type $\mathrm{GaP}$ grown under stoichiometric conditions, as shown in
Fig. 4. In Ga-rich material $\mathrm{V}_{\mathrm{Ga}}^{-3}$ is more expensive to form than $\mathrm{Ga}_{\mathrm{P}}$ and it will not be the most common type of defect for any value of the Fermi-level in the bandgap. On the other hand, in strongly $n$-type GaP the formation energy is still low enough to lead to a noticeable concentration of Ga vacancies. In $\mathrm{GaP}$ grown under P-rich conditions $\mathrm{V}_{\mathrm{Ga}}$ is more energetically favorable than in stoichiometric material and in the $n$-type region it should have its absolutely highest concentration, which is in fact the highest of all the defects being considered, irrespective of growth conditions and Fermilevel position. This is readily seen from a comparison of Figs. 5 and 6.

Isolated $\mathrm{V}_{\mathrm{Ga}}$ have been observed using EPR by Kennedy et al., but only after electron irradiation of the sample., ${ }^{4,5}$ According to them, although the sample is initially $p$-type the irradiation moves the Fermi level to a midgap position. In $p$-type and undoped $\mathrm{GaP}$ the best agreement was found by matching $\mathrm{V}_{\mathrm{Ga}}^{-2}$ with the data. The fact that this charge state was only seen after electron irradiation was taken as an indication that this is not the equilibrium charge state in semiinsulating $\mathrm{GaP}$.

In the first experiment ${ }^{4}$ the observed EPR data was interpreted as arising from an unpaired electron in a singlet state forming a spin $1 / 2$ defect center surrounded by four $\mathrm{P}$ atoms, which corresponds to a doubly negative charge state. This attribution of the signal to the singlet state was mainly due to the high symmetry of the observed experimental data for the defect; a triplet state with an odd number of electrons would be expected to be unstable to Jahn-Teller distortions. Since Kennedy and Wilsey observed a highly symmetric defect center it was assumed that the unpaired electron occupied the $A_{1}$ state of the vacancy, and that the $T_{2}$ and $A_{1}$ should have a reverse ordering.

Scheffler et al..$^{40}$ objected to this reverse ordering on theoretical grounds and claimed that their results contradicted the identification of the Ga vacancy in the doubly negative charge state $\mathrm{V}_{\mathrm{Ga}}^{-2}$. After this theoretical objection a new measurement ${ }^{5}$ then reassigned the EPR active defect state to a spin $3 / 2$ center corresponding to $\mathrm{V}_{\mathrm{Ga}}^{0}$. (It was suggested that the exchange coupling creates a ${ }^{4} A_{2}$ many-electron orbital singlet state which would be stable against Jahn-Teller distortions and hence in accordance with the experiments.)

Firstly, the conclusion that the -2 charge state is not the equilibrium state is in agreement with Fig. 4. In semiinsulating $\mathrm{GaP}$ the formation energy of $\mathrm{V}_{\mathrm{Ga}}^{-2}$ is approximately $0.5 \mathrm{eV}$ higher than that of $\mathrm{V}_{\mathrm{Ga}}^{-3}$. The more stable -3 charge state corresponds to all three $T_{2}$ levels being fully occupied and therefore it cannot be observed using EPR, while $\mathrm{V}_{\mathrm{Ga}}^{0}$ and $\mathrm{V}_{\mathrm{Ga}}^{-2}$ can.

Secondly, both of the EPR active states $\mathrm{V}_{\mathrm{Ga}}^{0}$ and $\mathrm{V}_{\mathrm{Ga}}^{-2}$ have structures which although Jahn-Teller distorted still lie very close to a perfect tetrahedron according to the results presented here. The question is whether the EPR experiments in question are sensitive enough to detect such a small deviation from the full tetragonal symmetry. If not then both the 0 and -2 charge states are possible candidates for the observed EPR defect. Except in strongly $p$-type $\mathrm{GaP}, \mathrm{V}_{\mathrm{Ga}}^{-2}$ will occur in much higher concentrations than the neutral vacancy, making it a much more likely candidate, although that alone is not enough to fully confirm this assignment. 
As previously stated, Krause-Rehberg only found positron trapping in $n$-type GaP. Reviewing this in the light of the results presented in this section we first note that $\mathrm{V}_{\mathrm{Ga}}$ has a very high formation energy in $p$-type material and therefore will not occur in a detectable concentration and hence not contribute to any positron trapping. In the experimental study the entire vacancy concentration responsible for the positron trapping in $n$-type $\mathrm{GaP}$ was attributed to negatively charged $\mathrm{V}_{\mathrm{P}}$. $\mathrm{V}_{\mathrm{Ga}}$ was incorrectly ruled out as a participant. The reason given being that $\mathrm{V}_{\mathrm{Ga}}$ occurs in the negative form throughout almost the entire bandgap, and no trapping was detected in $p$-type and semi-insulating material. Hence, it was concluded that the $\mathrm{V}_{\mathrm{Ga}}$ concentration must be vanishingly small. This argument does not take the linear dependence of the formation energy on the Fermi level into account. From Fig. 5 it is obvious that $\mathrm{V}_{\mathrm{Ga}}^{-3}$ will not occur in observable concentration in $p$-type material but that the concentration is very large under $n$-type conditions, even larger than the $\mathrm{V}_{\mathrm{P}}$ concentration. We therefore conclude that $\mathrm{V}_{\mathrm{Ga}}^{-3}$ cannot be excluded on these grounds but is instead responsible for the majority of the positron trapping in $n$-type GaP, although not for the charge transfer level which remains assigned to $\mathrm{V}_{\mathrm{P}}$.

$\mathrm{V}_{\mathrm{Ga}}$ in $\mathrm{GaP}$ has also been mentioned in connection to $\mathrm{Ga}$ self-diffusion where it was concluded that the diffusion is primarily mediated through one type of simple native defect. ${ }^{41}$ Parallels were then drawn to the case of GaAs, where it has been verified that Ga self-diffusion is mainly due to $\mathrm{V}_{\mathrm{Ga}}^{-3}$. The results presented here support this suggestion, the relatively high concentration of $\mathrm{V}_{\mathrm{Ga}}^{-3}$ in semiinsulating and especially $n$-type GaP makes it likely that the $\mathrm{V}_{\mathrm{Ga}}^{-3}$ defect mediates the $\mathrm{Ga}$ self-diffusion in $\mathrm{GaP}$ as well, as was suspected by Wang et al. ${ }^{42}$

\section{Gallium interstitial}

In this study, the formation energies of the interstitial defects have been calculated at both tetragonal interstitial sites: the phosphorus surrounded site and the gallium surrounded site. The two are not equivalent. $\mathrm{Ga}_{i: \mathrm{Ga}}^{0}$ has a fully occupied shallow $A_{1}$ defect level in the lower part of the bandgap and a singly occupied $T_{2}$ level just above the conduction band edge, whereas for $\mathrm{Ga}_{i: \mathrm{P}}^{0}$ the $A_{1}$ level is not as shallow and the $T_{2}$ level is instead located below the conduction band edge (Fig. 2). The difference is, of course, due to the different types of bond possible between the interstitial and its host neighbors (metallic and partially polarized covalent). Introducing the interstitial in pure bulk $\mathrm{GaP}$ causes strain which forces the surrounding crystal to relax outwards (Table I).

For $\mathrm{Ga}_{i: \mathrm{P}}^{+3}$ the nearest-neighbor distance changes to $2.36 \AA$ from the ideal distance of $2.33 \AA$ and the second nearest neighbors are also moved outwards by $0.15 \AA$. This corresponds to a defect volume of $6.76 \AA^{3}$ and an outward relaxation of $3.7 \%$. The +3 charge state leaves no localized electrons at the defect center and it is simply an unbonded ion at the interstitial site. For all charge states the full $T_{d}$ symmetry is maintained after relaxation.

The corresponding outward relaxations for $\mathrm{Ga}_{i: \mathrm{P}}^{+2}$ and $\mathrm{Ga}_{i: \mathrm{P}}^{+1}$ are 0.06 and $0.19 \AA$ for the nearest neighbors and 0.13 and
$0.05 \AA$ for the second nearest neighbors (giving volume increments of 7.1 and $11.5 \%$, respectively). The +1 state, which imposes the largest relaxation on the four nearest atoms but leaves all other atoms close to the ideal crystal positions, is the most stable configuration for all values of the Fermi level except in $p$-type GaP (Fig. 4). In $p$-type material the formation energy is, on the other hand, much lower for the +3 state than the +1 state, closely followed by the +2 charge state. This results in two levels $(+3 \mid+2)$ and $(+2 \mid+)$ in the lower part of the bandgap. $\mathrm{Ga}_{i: \mathrm{P}}^{+3}$ is therefore expected to have its highest concentration in $p$-type GaP.

$\mathrm{Ga}_{i: \mathrm{Ga}}$ is most stable in the singly positive charge state for all values of the Fermi level in the bandgap. The structure is very similar to that of the P-surrounded interstitial but the relaxation is somewhat larger, nearest neighbors are moved outwards by $0.21 \AA$. The formation energy is slightly lower than that of the P-surrounded interstitial.

In $\mathrm{GaP}$ grown under P-rich conditions $\mathrm{Ga}$ interstitials will hardly be observable at all (Fig. 5). In Ga-rich material on the other hand, the phosphorus surrounded Ga interstitial is more favorable and in the +3 charge state it will be the native defect that occurs in the highest concentration in strongly p-type GaP (Fig. 6).

The experimental observation of $\mathrm{Ga}_{i}$ has been reported by Chen and Monemar. ${ }^{14}$ Both $n$ - and $p$-type materials were used and analyzed using ODMR. The Ga interstitial was believed to be in a doubly positive charge state $\mathrm{Ga}_{i}^{+2}$ and to be bonded to another as yet unidentified negative defect. It is reasonable to expect the +2 charge state since that is the only magnetically active one, even though $\mathrm{Ga}_{i}^{+3}$ is here predicted to have a higher concentration in $p$-type GaP (Fig. 4). $\mathrm{Ga}_{i}^{+3}$ and $\mathrm{Ga}_{i}^{+1}$ are not magnetically active can therefore not be observed. Surprisingly, the strongest ODMR signal was found in $n$-type material which must be due to $\mathrm{Ga}_{i}$ not being in its isolated form.

$\mathrm{Ga}_{i}$ has also been observed ${ }^{42}$ in $\mathrm{Ga}_{0.98} \mathrm{Al}_{0.02} \mathrm{~N}_{0.01} \mathrm{P}_{0.99}$, where two different configurations were identified. These were both at $T_{d}$ sites surrounded by either group-III or group- $\mathrm{V}$ atoms. This is agreement with the results presented here. The two interstitial sites are expected to be almost equally common because of the small difference in formation energy (Fig. 3). Furthermore, at both sites the interstitial is found to relax back to the tetragonal structure when distorted (see Table I).

\section{Phosphorus interstitial}

Computationally, the phosphorus interstitial is treated in the same way as the gallium interstitial. The relaxation effects are much larger though, resulting in a singly occupied defect level now at midgap for the neutral interstitial, which gives a qualitatively different behavior for the charge transfer levels. For $\mathrm{P}_{i: \mathrm{P}}^{+3}$ the nearest neighbors are practically at the ideal atom distances for the tetragonal interstitial site (they deviate by only $0.03 \AA$ ) but the second nearest neighbors are all moved outwards by $0.17 \AA$. The +3 charge state has the lowest formation energy, which is reached in strongly $p$-type $\mathrm{GaP}$. The lowest value of this formation energy is $2.5 \mathrm{eV}$ under P-rich conditions, so not even the lowest formation 
energy of the $\mathrm{P}_{i}$ will correspond to a noticeable concentration (see the right scale in Fig. 5). The other charge states give a stronger bonding of the interstitial atom to the surrounding lattice and a greater relaxation of the surrounding structure with increasing numbers of electrons. Since this does not bring the formation energies down to interesting values these structures will not be described in detail. Experimental observation of $\mathrm{P}_{i}$ has never been reported in the literature, and from the high formation energies in Fig. 4 this is to be expected. Indeed, the phosphorus interstitial has the highest formation energy of all the native defects. So under equilibrium conditions it will not be present in any detectable concentrations under any of the circumstances presented here.

\section{E. Phosphorus antisite}

The $\mathrm{P}$ antisite has a deep $A_{1}$ level in the bandgap, as shown for the neutral case in Fig. 2. $\mathrm{P}_{\mathrm{Ga}}$ is therefore a possible center for nonradiative recombination. This possibility was investigated by Killoran et al. who concluded that it was indeed the case. ${ }^{12}$ For the $\mathrm{P}_{\mathrm{Ga}}^{+2}$ state this deep level is unoccupied. Since phosphorus has five valence electrons and gallium three, the $s p^{3}$ bonding structure of the host material can be preserved in this charge state. The perfect tetragonal symmetry can then be maintained, as shown in Table I. (This is also expected for the other two charge states since this defect is not Jahn-Teller active.) All four nearest-neighbor distances of $\mathrm{P}_{\mathrm{Ga}}^{+2}$ are therefore $2.23 \AA$. This reduction can be explained by the smaller covalent radius of phosphorus. The sum of the covalent radii for $\mathrm{P}$ and $\mathrm{Ga}$ is $2.31 \AA$ which is very close to the $\mathrm{GaP} s p^{3}$ bonding distance of $2.33 \AA$ while twice the covalent radius of $\mathrm{P}$ is equal to $2.20 \AA$, indicating that $\mathrm{P}_{\mathrm{Ga}}^{+2}$ is locally similar to a phosphorus $s p^{3}$ structure. This gives an inward relaxation of $4.3 \%$ compared to the Ga-P bonding distance in defect free $\mathrm{GaP}$ and reduces the nearest-neighbor volume by $12.6 \%$.

In the singly positive charge state $\mathrm{P}_{\mathrm{Ga}}^{+1}$, one additional electron is introduced in the deep level. The structure is still of full tetragonal symmetry but the inward relaxation is smaller so the defect volume is only changed by $3.2 \%$ compared to the ideal lattice. The effect is also smaller in terms of energy, the formation energy is lowered by only $0.07 \mathrm{eV}$ upon relaxation (Table I).

In the neutral case the $\mathrm{P}$ atom contributes all five of its valence electrons. The structure again relaxes back to the full $T_{d}$ symmetric structure if distorted, and the nearest-neighbor distances increase from 2.33 to $2.39 \AA$. In contrast to the other two charge states this gives an outward relaxation and a defect volume $7.4 \%$ larger than for the ideal antisite.

From Fig. 4 it is seen that $\mathrm{P}_{\mathrm{Ga}}^{+2}$ is the energetically favored charge state in $p$-type material and in $n$-type material the $\mathrm{P}_{\mathrm{Ga}}^{0}$ state has the lowest formation energy. This confirms the expectation that the antisite acts as a deep double donor. This has been shown experimentally ${ }^{3}$ in $p$-type $\mathrm{GaP}$, since the EPR active singly positive charge state was not seen without optical excitation which was taken as an indication that the equilibrium charge state is the doubly positive one.

According to our results the $\mathrm{P}_{\mathrm{Ga}}^{+1}$ state is, in fact, never the most stable. Although the antisite structure is a fully $T_{d}$ sym- metric $s p^{3}$-bonded tetrahedron for all three charge states, the strong change of defect volume causes a reverse ordering of the charge transfer levels. Since the nearest-neighbor volume increases drastically going to the neutral charge state the electron-electron repulsion is reduced so that it becomes energetically favorable for the $\mathrm{P}$ antisite to go straight from the unoccupied $A_{1}$ level of the $\mathrm{P}_{\mathrm{Ga}}^{+2}$ state to the fully occupied $A_{1}$ level of the neutral $\mathrm{P}_{\mathrm{Ga}}^{0}$ state. This puts the $(+\mid 0)$ charge transfer level below the $(+2 \mid+)$ level, leaving only a $(+2 \mid 0)$ level.

Regarding the stoichiometry, $\mathrm{P}_{\mathrm{Ga}}$ should be favored if there is more phosphorus present than gallium during the growth of the crystal. This can be seen from a comparison of the formation energies in $\mathrm{GaP}$ under stoichiometric, $\mathrm{P}$ - and Ga-rich conditions, shown in Figs. 4-6. The difference in formation energy for $\mathrm{P}_{\mathrm{Ga}}$ between Ga-rich and P-rich $\mathrm{GaP}$ is $1.75 \mathrm{eV}$ which corresponds to a huge difference in the defect concentration.

Considering the relative concentrations in stoichiometric material, the doubly positive charge state of the phosphorus antisite $\mathrm{P}_{\mathrm{Ga}}^{+2}$ is the most common defect of all for values of the Fermi level in the lower half of the bandgap.

In $\mathrm{GaP}$ grown under Ga-rich conditions $\mathrm{P}_{\mathrm{Ga}}$ is energetically very expensive, so that it is never being the most stable native defect, for any value of the Fermi level. The defect concentration is much lower than in stoichiometric material and there will only be moderate concentrations in strongly p-type material, at best.

In P-rich $\mathrm{GaP}$ the $\mathrm{P}_{\mathrm{Ga}}^{+2}$ antisite occurs with the highest concentration of any native defect in both semi-insulating and $p$-type GaP. The dependence of the defect concentration on the formation energy for the doubly positive charge state means that the closer the Fermi level is to the valence band the higher the $\mathrm{P}_{\mathrm{Ga}}^{+2}$ concentration will be. In $n$-type material the antisite therefore occurs in much lower concentrations, in the form of the neutral charge state.

In experiments, $\mathrm{P}_{\mathrm{Ga}}$ is the most clearly identified defect to date. This suggests that there are significant amounts of it present. Its occurrence was first found through EPR measurements in semi-insulating $\mathrm{GaP}$ (Ref. 1) where it was thought to be in a singly positive charge state and with tetragonal symmetry. Here the $\mathrm{P}_{\mathrm{Ga}}^{+1}$ structure is found to be tetragonal within an error of $1.35 \%$, i.e., the maximal deviation of any of the four nearest neighbour distances is $1.35 \%$ of the average distance. These EPR results were made in GaP grown under P-rich conditions, under which a high $\mathrm{P}_{\mathrm{Ga}}$ concentration would indeed be expected according to Fig. 5. The limitation of EPR is that only unpaired electrons and paramagnetic states can be detected and therefore only certain charge states of the defect can be observed. In the case of the P antisite the $\mathrm{P}_{\mathrm{Ga}}^{+1}$ state is the only EPR active one.

For this charge state Kaufmann et al. found a concentration of $4 \times 10^{16} \mathrm{~cm}^{-3}$ in $p$-type $\mathrm{GaP}$ but of less than $10^{15} \mathrm{~cm}^{-3}$ in $n$-type material. ${ }^{3}$ In $p$-type GaP the EPR active singly positive charge state was only detected after optical excitation by $1.25 \pm 0.10 \mathrm{eV}$. This was taken as an indication that $\mathrm{P}_{\mathrm{Ga}}^{+2}$ is the equilibrium charge state for values of the Fermi level close to the valence band, rather than $\mathrm{P}_{\mathrm{Ga}}^{+2}$. This optical ionization energy was ascribed to the $(+++2)$ charge 
transfer level at a location of $1.25 \pm 0.10 \mathrm{eV}$ above the valence band.

The relative size of these concentrations is in good agreement with our results, since the low formation energy of the $\mathrm{P}_{\mathrm{Ga}}^{+2}$ state in $p$-type material would yield a higher concentration of singly positive antisites after optical excitation than the neutral antisite in $n$-type GaP. Comparing these experimental results to Fig. 4, the $(++2)$ transfer level is calculated to lie $0.97 \mathrm{eV}$ above the valence band, the value being to low due to the LDA error. Considering the LDA error this is actually in very good agreement with the experimental value of $1.25 \mathrm{eV}$. Furthermore, the $(0 \mid+)$ is calculated to be at $0.93 \mathrm{eV}$. There will therefore be a reverse ordering of these levels and the only actual charge transfer level will be the $(0 \mid+2)$ level. Since $\mathrm{P}_{\mathrm{Ga}}^{+1}$ is the only EPR active state the experimental value of $1.25 \mathrm{eV}$ must still be attributed to the $(+\mid+2)$ level even though the $\mathrm{P}_{\mathrm{Ga}}^{+1}$ state is never the most stable.

However, in semi-insulating material with the Fermi level around midgap, $\mathrm{P}_{\mathrm{Ga}}^{+1}$ was observed without optical excitation. ${ }^{3}$ This is in accordance with the results presented here since the formation energy of the +1 charge state is only slightly larger than that of the most stable charge state in semi-insulating GaP. It therefore could well be present in considerable amounts even without excitation other than the thermal excitation corresponding to room temperature.

It was also claimed ${ }^{3}$ that there should be a second higher charge transfer level, namely the $(0 \mid+)$ level, at somewhere less than $0.8 \mathrm{eV}$ from the conduction band. This was expected because of a private communication from Scheffler $e t$ al., whose unpublished paper was referred to. Later, when published, their work strengthened the identification of $\mathrm{P}_{\mathrm{Ga}}$ through semiempirical self-consistent Green's-function calculations. The $(0 \mid+)$ and $(+\mid+2)$ charge transfer levels were calculated to be 0.6 and $1.1 \mathrm{eV}$ from the conduction band, respectively. (This corresponds to 1.75 and $1.25 \mathrm{eV}$ from the valence band and the good agreement of the second level arises because the pseudopotentials were adjusted to recover the experimentally measured bandgap.). ${ }^{40,43}$ The higher $(0 \mid+)$ level was claimed by Scheffler et al. to be in good agreement with the experimental work of Kaufmann et al., although no experimental evidence for a second higher level was ever actually presented. The study of Scheffler et al. did not suffer from finite size errors, but at the same time did not take relaxations into account and therefore any reverse ordering of the charge transfer levels would not be detected. So far there exists no experimental evidence for this second, higher charge transfer level for $\mathrm{P}_{\mathrm{Ga}}$ which would contradict the single $(+2 \mid 0)$ level found in this work. ${ }^{3}$ However, it cannot be excluded that the reverse ordering found here is a consequence of the LDA error. (Finite-size errors can be excluded as shown in Sec. IV).

\section{F. Gallium antisite}

The Ga antisite has a $T_{2}$ level in the bandgap. For the neutral case this is split into two degenerate and fully occupied defect levels and one higher empty defect level, all in the lower half of the bandgap. In the doubly negative charge state $\mathrm{Ga}_{\mathrm{P}}^{-2}$, all three defect levels are fully occupied so no symmetry breaking is expected to occur. In this charge state the antisite atom has an equal number of valence electrons as the $\mathrm{P}$ atom it is substituting. Thus the $s p^{3}$ bonding structure is maintained and the relaxed structure has full tetragonal symmetry. After relaxation the bonding distance to the four surrounding Ga atoms is $2.30 \AA$ for all four bonds, an inward relaxation of $1.3 \%$ of the ideal Ga-P bond length or $4.3 \%$ of the volume.

In the $\mathrm{Ga}_{\mathrm{P}}^{-1}$ state the $T_{d}$ symmetry is broken, reduced to $C_{2 v}$, since the $\mathrm{Ga}$ antisite atom bonds more strongly to two of its nearest neighbors located at distances of $2.31 \AA$. (The antisite atom at the same time pulls the opposite two nearest neighbors causing their neighbor-neighbor distance to be reduced to $3.70 \AA$ in comparison to, on average, $3.85 \AA$ for the remaining five neighbor-neighbor distances). The other two nearestneighbors of the antisite are located $2.38 \AA$ away, increasing the defect volume slightly, by $0.8 \%$.

This outward relaxation increases with more positive charge states, being $5.4 \%$ for $\mathrm{Ga}_{\mathrm{p}}^{0}$. For this charge state the $\mathrm{Ga}$ antisite atom is located in the middle of the tetrahedron with equal distances of $2.38 \AA$ to all nearestneighbors (to an accuracy of $0.2 \%$ ). In spite of this, the structure does not have full $T_{d}$ symmetry but $D_{2 d}$ symmetry, since rotation of some neighbors around the antisite makes two opposite sides of the nearest-neighbor tetrahedron noticeably longer than the rest, i.e., two neighbour-neighbor distances are equal to $4.05 \AA$ instead of $3.81 \AA$ as for the other four.

For the $\mathrm{Ga}_{\mathrm{P}}^{+1}$ charge state the nearest-neighbor tetrahedron is the same as for the neutral structure, although the four short distances are somewhat larger, $3.91 \AA$ rather than $3.81 \AA$, resulting in a $11.5 \%$ outward relaxation for the volume. The largest change is that the Ga antisite atom moves away from the middle of the tetrahedron (as for the -1 charge state), now with two short distances of $2.37 \AA$ and two longer distances of $2.47 \AA$, to the nearestneighbors, lowering the symmetry further from $D_{2 d}$ to $C_{2 v}$ (see Table I).

For the last stable charge state, the doubly positive $\mathrm{Ga}_{\mathrm{P}}^{+2}$, the relaxation takes the structure back to the high symmetry $T_{d}$ configuration, with the four antisite to nearest-neighbor distances equal to $2.45 \AA$ and the six neighbor-neighbor distances equal to $4.00 \AA$. This results in a vacancy volume of $7.57 \AA^{3}, 16.1 \%$ larger than the ideal volume: the largest of all the charge states.

Khoo and Ong also studied the Ga antisite using the cluster method described earlier. ${ }^{22}$ They only included relaxations for the $\mathrm{Ga}$ antisite atom and its nearest-neighbors. Since $\mathrm{Ga}_{\mathrm{P}}$ is expected to act similar to a double acceptor, they examined only it's neutral, singly and doubly negative charge states. They found that the full tetrahedral symmetry was maintained for the doubly negative charge state $\mathrm{Ga}_{\mathrm{P}}^{-2}$ and that the Ga nearest-neighbors relax radially outwards by $0.19 \AA$. (This is close to the difference in atomic radii between Ga and P.) This means that the symmetry group of the $\mathrm{Ga}_{\mathrm{P}}^{-2}$ state is in agreement with that found here, but the large outward relaxation disagrees with the small inward relaxation found here.

They also found that the relaxation for $\mathrm{Ga}_{\mathrm{p}}^{-1}$ was exactly the same as for the doubly negative state, which is not supported by this study, where distinctly different structures are 
found. In the cluster calculations $\mathrm{Ga}_{\mathrm{P}}^{0}$ underwent a large symmetry breaking in which one of the antisite to nearestneighbor distances was elongated more than the other three, resulting in a $21 \%$ increase in comparison to the ideal bond length. Again, this is in disagreement with our results were the antisite atom is at an equal distance from all nearest neighbors.

These discrepancies are believed to be a consequence of either the very restricted relaxation used or simply a failure to fully converging the relaxations in the cluster calculations. [A similar structure for the $\mathrm{Ga}_{\mathrm{P}}^{0}$ was actually found in our preliminary calculations using only $\mathbf{k}=\left(\frac{1}{4}, \frac{1}{4}, \frac{1}{4}\right)$ and lower convergence criteria, for example. However, it proved to be unstable when a larger $k$-point grid and a higher convergence criteria were introduced.]

From the formation energies in Fig. 4 it is seen that $\mathrm{Ga}_{\mathrm{P}}^{+2}$ is the most stable state for the Ga antisite in strongly $p$-type $\mathrm{GaP}$, followed by the $\mathrm{Ga}_{\mathrm{P}}^{0}$ and $\mathrm{Ga}_{\mathrm{P}}^{-1}$ states over short intervals. $\mathrm{Ga}_{\mathrm{P}}^{-2}$ is the dominant charge state in everything but strongly $p$-type material. So for the most part $\mathrm{Ga}_{\mathrm{P}}$ does indeed act similar to a double acceptor. [In the last section it will be shown that extrapolating the results to an infinite supercell size leaves only a $(0 \mid-2)$ charge transfer level.] The difference in formation energy between the $n$ - and $p$-type regions is around $1.5 \mathrm{eV}$. This corresponds to a large difference in the order of magnitude for the concentrations. In stoichiometric material $\mathrm{Ga}_{\mathrm{p}}$ will therefore only occur in relevant amounts in semi-insulating and $n$-type material where it occurs in the -2 charge state. It is the dominant native defect in semi-insulating GaP but its density is considerably larger in $n$-type material.

Since the antisite defects replace one type of atom with the other type their formation energies undergo the largest change of all energy when going from $\mathrm{P}$ - to Ga-rich conditions. Because of this large variation $\mathrm{Ga}_{\mathrm{P}}$ will not occur in any significant amount in P-rich $\mathrm{GaP}$ whereas it will be the main native defect in Ga-rich $\mathrm{GaP}$ in the form of $\mathrm{Ga}_{\mathrm{P}}^{-2}$ (Figs. 4-6).

Khoo and Ong found a negative-U (0|2-) charge transfer level for $\mathrm{Ga}_{\mathrm{P}}$ in contrast to the two closely located charge transfer levels $(0 \mid-)$ and $(-\mid-2)$ presented in this report. This is an effect of the limiting supercell size as will be shown in Sec. IV.

Experimentally, $\mathrm{Ga}_{\mathrm{P}}$ has only been observed using ESR after neutron irradiation of the GaP sample. ${ }^{6}$ It was suggested that it was part of a defect complex and not in the isolated form. From the results presented here, $\mathrm{Ga}_{\mathrm{p}}^{-2}$ is predicted to be the most stable native defect of all in semi-insulating stoichiometric $\mathrm{GaP}$, but it will not be visible to ESR since all defect levels are fully occupied. The formation energies of the other charge states are so much higher that they will hardly be visible without some form of excitation, specially in a sample grown under P-rich conditions as in the experiment. In $\mathrm{GaP}$ grown under Ga-rich conditions however, $\mathrm{Ga}_{\mathrm{P}}$ is the dominant native defect throughout the entire bandgap. Here, even ESR active charge states may occur in concentrations high enough to be detected by ESR with little or no irradiation.

\section{DISCUSSION AND CONCLUSIONS}

To show the magnitude of the relaxation for each of the defects and also for the sake of comparing to older studies which do not include relaxations, the formation energies of the unrelaxed defects are presented in Fig. 3. Comparing with Fig. 4, it can be seen that the interstitials, which impose large stresses on the surrounding crystal, generally gain the most from relaxation. Qualitatively, these unrelaxed results compare well with the results of Jansen and Sankey. ${ }^{25}$ The formation energies for $\mathrm{V}_{\mathrm{Ga}}$ and $\mathrm{P}_{\mathrm{Ga}}$ agree to within $0.5 \mathrm{eV}$ but our energies for the interstitials are considerably lower. These differences can be ascribed to their use of an rather small basis set and a small 32 atom supercell, leading to a mixing of even the deep levels with the conduction band, as they themselves point out. It is therefore not very meaningful to make exact comparisons of the formation energies or charge transfer levels, although the most stable charge state as a function of the Fermi-level for each defect agrees well.

Since all the native defects in this study can occur at an equal number of possible lattice sites, the defect formation energies can be related directly to defect concentrations in Figs. 4-6. This estimate is calculated for $300 \mathrm{~K}$ using an approximate formation entropy contribution of $5 k_{B}$ for all defects. This is not intended to be interpreted as an exact value but to give the reader an idea of the rapid variation in the order of magnitude of the defect concentrations. From this it is concluded that defects with formation energies around and above $1.5 \mathrm{eV}$ will simply not be present in realistic quantities at thermodynamic equilibrium at room temperature. The defects that will occur in concentrations large enough to influence the electronic properties of the semiconductor have formation energies below $1 \mathrm{eV}$.

In stoichiometric $\mathrm{GaP}$ these are the $\mathrm{P}_{\mathrm{Ga}_{2}}^{+2}$ in $p$-type material and in $n$-type primarily $\mathrm{V}_{\mathrm{Ga}}^{-3}$, although $\mathrm{Ga}_{\mathrm{P}}^{-2}$ will also occur in measurable quantities. $\left(\mathrm{Ga}_{\mathrm{p}}^{-2}\right.$ will also be the most common native defect for values of the Fermilevel in the middle of the bandgap, although the concentration is vanishingly small in comparison to that in $n$-type material).

In $\mathrm{GaP}$ grown under P-rich conditions $\mathrm{P}_{\mathrm{Ga}}$ is again the most common defect in $p$-type material, but the concentration increases by 15 orders of magnitude in comparison to the stoichiometric case. $\mathrm{V}_{\mathrm{Ga}}^{-3}$ is also more favorable in P-rich material and it is the dominant defect in $n$-type GaP. Its concentration is increased by 7 orders of magnitude in comparison to the stoichiometric material. The concentrations of $\mathrm{P}_{\mathrm{Ga}}^{+2}$ and $\mathrm{V}_{\mathrm{Ga}}^{-3}$ in, respectively, $p$ - and $n$-type P-rich material, are predicted to be among the highest intrinsic defect concentrations in $\mathrm{GaP}$.

In $\mathrm{Ga}$ rich $\mathrm{GaP}$ no defect will reach a high concentration in the $p$-doped region, according to the above criterion, but in the range from semi-insulating to strongly $n$-type material $\mathrm{Ga}_{\mathrm{p}}^{-2}$ will have a substantial concentration. Although this antisite is the dominant defect, both types of vacancies, in the form of $\mathrm{V}_{\mathrm{Ga}}^{-3}$ and $\mathrm{V}_{\mathrm{P}}^{-5}$, will also occur at considerable concentrations in strongly $n$-type GaP. (The most common defect in the $p$-type region will be $\mathrm{Ga}_{i: \mathrm{P}}^{+3}$ although its formation energy is larger than $1 \mathrm{eV}$.)

The presence of native defects will restrict the range of Fermi-level positions one can achieve with doping. GaP 
grown under P-rich conditions (Fig. 5) will be hard to make extremely $n$-type ( $p$-type), for example; since placing the Fermi level close to the conduction band (valence band) makes it favorable to form a large concentration of $\mathrm{V}_{\mathrm{Ga}}^{-3}\left(\mathrm{P}_{\mathrm{Ga}}^{+2}\right)$ which decreases the number of negative (positive) charge carriers, therefore lowering (raising) the Fermi-level position: a process known as compensation. From this it is concluded that in order to produce $\mathrm{GaP}$ with as strong $p$-type doping as possible it would be advantageous to do so in a $\mathrm{GaP}$ sample grown under Ga-rich conditions, since then no native defect has a particularly high concentration in the p-type region (Fig. 6).

As written earlier in the Results Section, the assertion that positron trapping in $\mathrm{GaP}$ in mainly due to $\mathrm{V}_{\mathrm{P}}$ defects is questioned here. Even under Ga-rich conditions (the most favorable for producing $\mathrm{V}_{\mathrm{P}}$ ), the concentration of $\mathrm{V}_{\mathrm{P}}$ is only on the order of $10^{9} \mathrm{~cm}^{-3}(T=300 \mathrm{~K})$. Even when taking the upper limit of the estimated formation entropy into account, $S_{f}=10 k_{B},{ }^{34,35}$ the concentration is only raised to $10^{13} \mathrm{~cm}^{-3}$. This is still well below the experimentally observed concentration of $10^{16} \mathrm{~cm}^{-3}$ in $n$-type $\mathrm{GaP} .^{17}$ There has been yet another positron lifetime experiment, which found a vacancy concentration on the order of $10^{17} \mathrm{~cm}^{-3}$, also in $n$-type GaP. ${ }^{16}$ From a comparison to a similar study in GaAs it was concluded that this concentration should be due to $V_{P}$. However, performing the estimation for $\mathrm{V}_{\mathrm{Ga}}$ using our data, one finds a concentration of $10^{18} \mathrm{~cm}^{-3}$ for $\mathrm{V}_{\mathrm{Ga}}$ in the -3 charge state in stoichiometric $\mathrm{GaP}$ under strongly $n$-type conditions $\left(E_{F}=\varepsilon_{c}\right)$. Considering that such a doping is a little extreme for realistic $\mathrm{GaP}$, and that there would also be a small contribution from the formation entropy, the two experimental values show an excellent agreement with this theoretical estimate. It is therefore concluded that, under thermodynamic equilibrium, $\mathrm{V}_{\mathrm{Ga}}$ and not $\mathrm{V}_{\mathrm{P}}$ should be responsible for the largest vacancy concentration in $n$-type $\mathrm{GaP}$ and hence for the positron trapping behavior.

As mentioned before, the largest sources of error in this study (besides those arising from the LDA itself) are the finite size errors due to the supercell approximation. To what degree these affect the results presented here has been studied using finite size scaling, extrapolating the formation energies to those of an infinite supercell size according to Refs. 39 and 40. There it was demonstrated that the leading error terms are inverse linear and inverse cubic in the supercell size L. Physically, this means that the errors scale predominantly with the defect-defect image distance and with the cell volume rather than with, say, the cell surface area (inverse square). This is because most of the quantities in the system which are related to the finite size errors (for example, the jellium charge density, the number of atoms and the number of electrons) scale with the volume. As a result, a reasonable estimate of the formation energy for an isolated defect can be obtained by extrapolating the calculated formation energies using the fitting equation

$$
E_{\mathrm{sc}}^{\mathrm{form}}(L)=E_{\infty}^{\mathrm{form}}+\frac{a_{1}}{L}+\frac{a_{3}}{L^{3}} .
$$

Here, $E_{\mathrm{sc}}^{\mathrm{form}}(L)$ is the formation energy as a function of the supercell size, $E_{\infty}^{\text {form }}$ is the extrapolated formation energy for an infinite supercell and $a_{1}$ and $a_{3}$ are fitting parameters. It was found ${ }^{37,44}$ that by using four supercell sizes up to 512 atoms it is possible to obtain $E_{\infty}^{\text {form }}$ values which are accurate to around $0.01-0.1 \mathrm{eV}$, for defects with charges up to about \pm 3 . Here, having only 3 points to fit (which is still reasonable since the expected form of the error scaling is known), we should anticipate slightly larger errors, around $0.1-0.3 \mathrm{eV}$.

The infinite limit corresponds to a completely isolated defect while one defect in a 216 atom supercell corresponds to an ordered defect array of concentration of $2 \times 10^{20} \mathrm{~cm}^{-3}$. The scaling curves are calculated from 8, 64, and 216 atom supercells and presented for only the most abundant native defects: the vacancies, the antisites and the gallium interstitial. The formation energies for the $\mathrm{P}_{i}$ increase going from the 64 to the 216 atom supercell, making it less common in the infinite limit and therefore it may be ignored.

For the $\mathrm{P}$ antisite the error from using a 216 atom supercell is small. As seen from Fig. 7, all three charge states would have a slightly larger formation energy in the limit of an infinite supercell size. This decreases the defect concentration somewhat, but leaves the relative stability of the charge states the same. The position of the charge transfer level is altered by less than $0.1 \mathrm{eV}$.

In the 216 atom supercell $\mathrm{Ga}_{\mathrm{P}}^{+2}$ the most stable charge state of the $\mathrm{Ga}$ antisite defect in $p$-type GaP. Extrapolating to the infinite limit in Fig. 7 it is seen that the formation energies of the positive charge states are increased relative to the neutral state and because of their linear dependence on the Fermi level they will therefore never be stable. The singly negative charge state is also less favorable in the infinite limit leaving only one negative-U $(0 \mid-2)$ charge transfer level inside the band gap (Fig. 8). Since the formation energy of the doubly negative charge state increases more than the neutral going to the infinite limit, this level is shifted up in energy within the bandgap by about $0.1 \mathrm{eV}$.

The $\mathrm{P}$ vacancy will also undergo an upward shift of the charge transfer levels going to the infinite supercell size limit. The formation energy of the singly positive charge state is lowered while it is unchanged for the -1 charge state resulting in the negative-U $(+\mid-)$ charge transfer level moving up in energy by approximately $0.1 \mathrm{eV}$. The formation energies of the -3 and -5 charge states are so much higher in the infinite limit that the higher charge transfer levels are both shifted out of the theoretical bandgap.

The formation energy of the +1 charge state of the $\mathrm{Ga}$ surrounded $\mathrm{Ga}$ interstitial remains essentially unchanged upon scaling (Fig. 7). For the P surrounded site all the charge states will move up in energy, making them less stable than the Ga surrounded site. Because of this and of the lowering in energy of $\mathrm{V}_{\mathrm{P}}^{+1}$, the $\mathrm{P}$ vacancy will become more stable than the $\mathrm{Ga}$ interstitial in $p$-type $\mathrm{GaP}$ in the infinite limit, in contrast to the results of the 216 atom supercell (Fig. 8).

For the Ga vacancy (Fig. 7) the largest effect of the scaling will be that the charge transfer levels shift up in energy, for the $(-2 \mid-3)$ level by as much as $0.2 \mathrm{eV}$, as shown in Fig. 8. The formation energy of the $\mathrm{V}_{\mathrm{Ga}}^{-3}$ state will also be raised by about $0.8 \mathrm{eV}$, lowering the defect concentration in the infinite limit correspondingly.

The predicted defect stabilities, extrapolating to an infinite supercell size, are summarized in Fig. 8. The effect of re- 


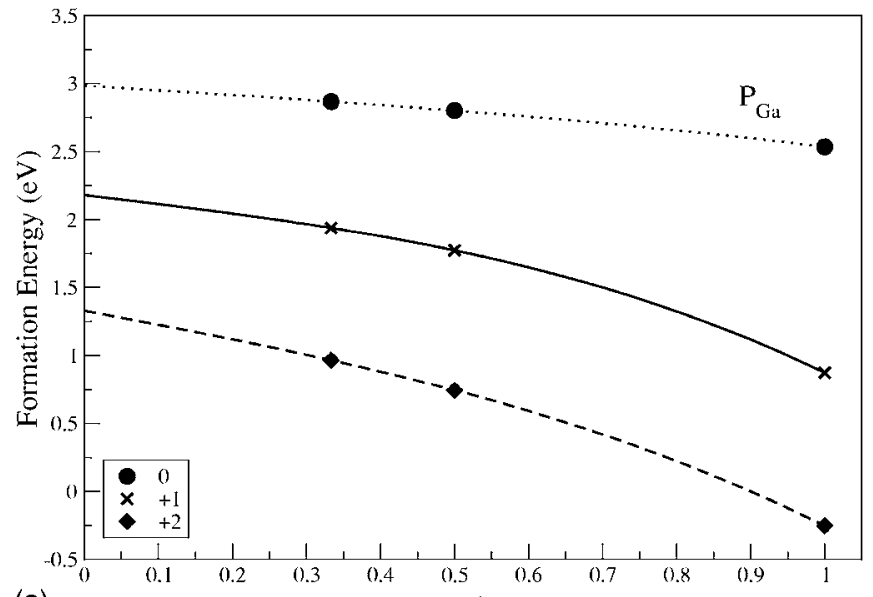

(a)

$1 / \mathrm{L}$

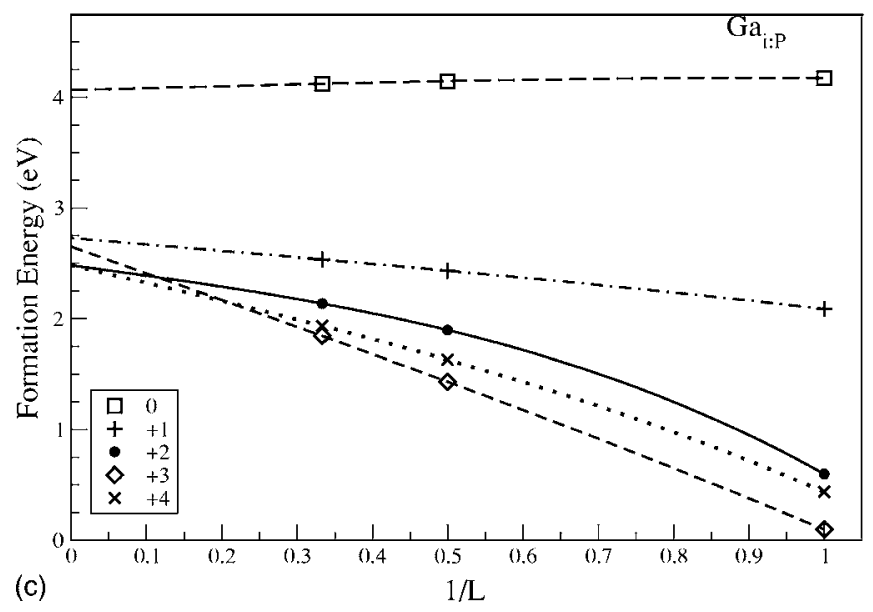

(c)

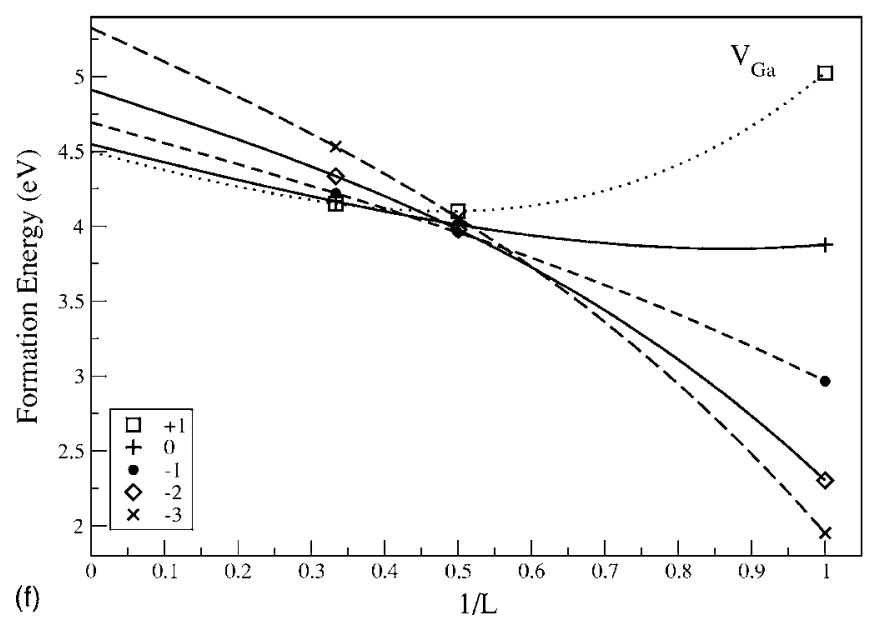

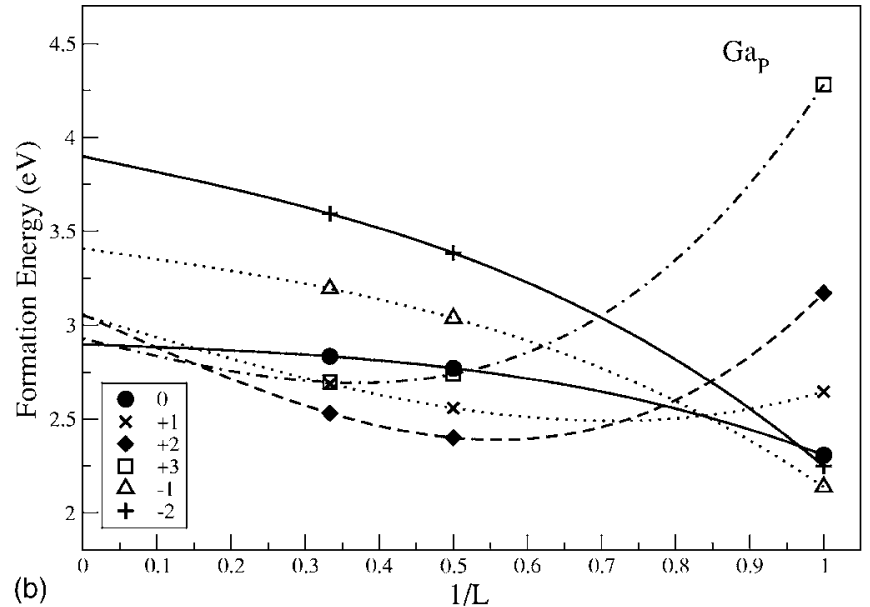

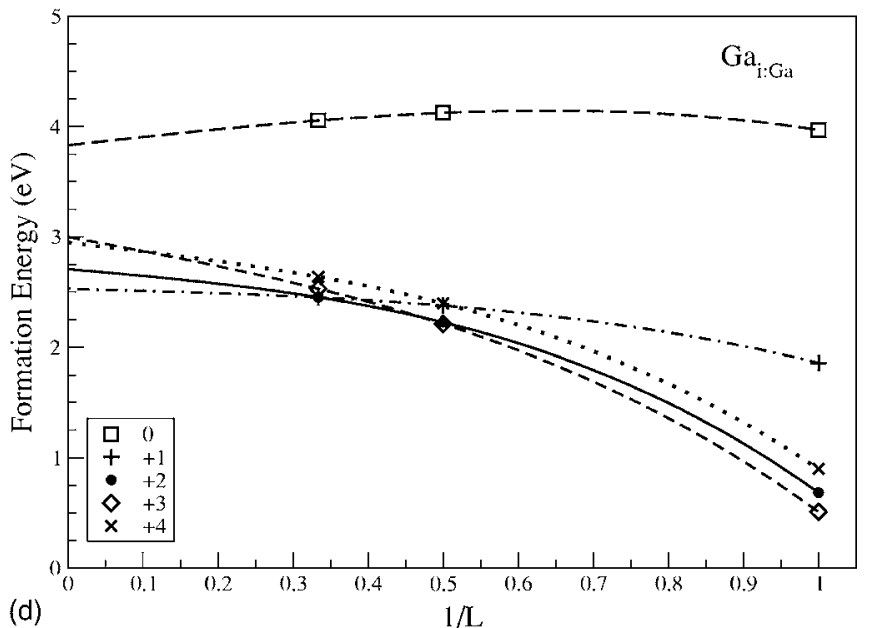

(d)

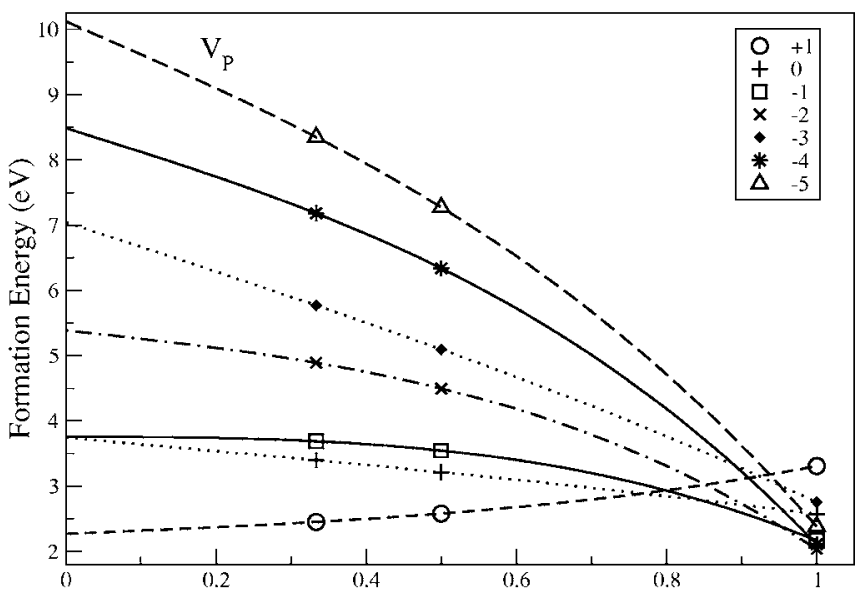

$1 / \mathrm{L}$

FIG. 7. Finite size scaling of formation energies for the most occurring defects. $L$ is the length of the supercell side in unit $s$ of the simple cubic 8 atom supercell side length, so that $1 / L=1,1 / L=\frac{1}{2}$, and $1 / L=\frac{1}{3}$ for the 8,64 , and 216 atom supercells, respectively.

moving finite size errors can be seen directly from a comparison to the grey curves in the background, which show the formation energies of the 216 supercell, as in Fig. 4. The positive charge states of $\mathrm{Ga}_{\mathrm{P}}$ as well as the -3 and -5 charge states of $\mathrm{V}_{\mathrm{P}}$ are seen to be an artifact of the limited size of the 216 atom supercell and disappear in the scaling. Apart from that, the scaling does not affect the the qualitative picture much although overall it has a tendency to decrease the predicted defect concentrations somewhat.

Regarding the comparison between experiments and our scaled results, for the most part little changes. Our interpretation of the EPR and ODMR measurements remains the 


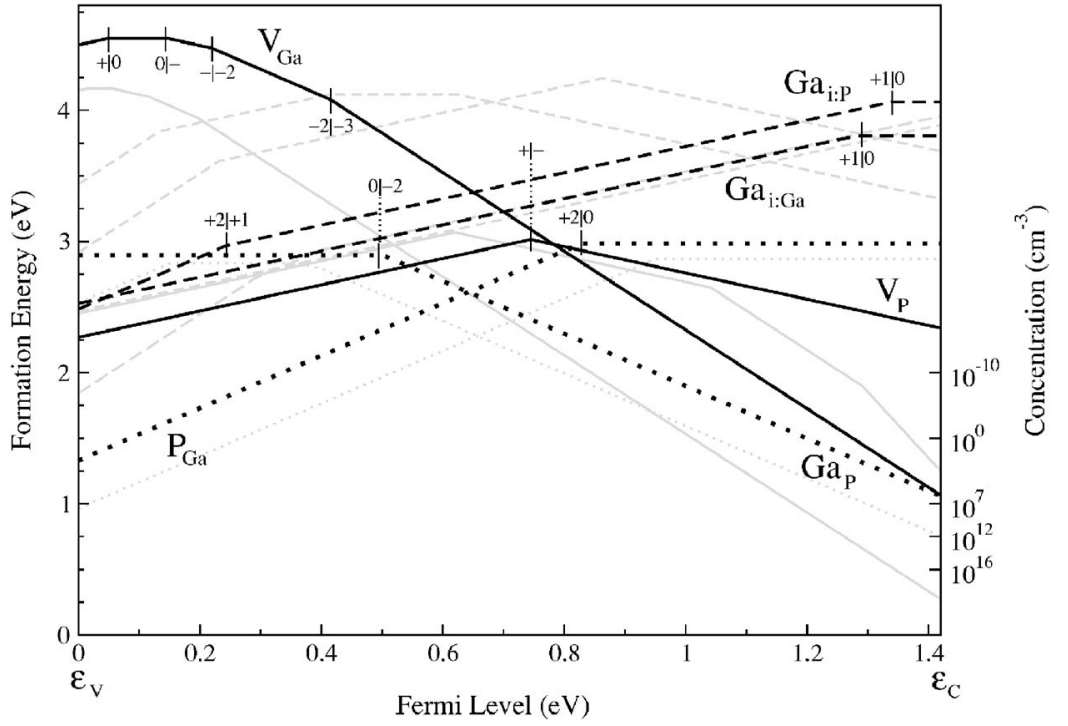

FIG. 8. The formation energies, extrapolated to the infinite supercell size limit, plotted as a function of the Fermi-level for all relaxed native defects under stoichiometric conditions $\left(\mu_{\mathrm{Ga}}=-4.06 \mathrm{eV}\right)$. The grey curves are shown for comparison and correspond to the results of the 216 atom supercell (as in Fig. 4). same, as does our central conclusion regarding positron annihilation, namely that it results mostly from $\mathrm{V}_{\mathrm{Ga}}$. However, the $(-\mid-3)$ and $(-3 \mid-5)$ charge transfer levels of $V_{P}$ are shown by scaling to actually lie outside the bandgap (about $0.17 \mathrm{eV}$ above). Since the $(-3 \mid-4)$ level of $\mathrm{V}_{\mathrm{Ga}}$ lies well above the bandgap also after scaling there is no obvious candidate for the charge transfer level believed to have been observed in positron annihilation experiments by KrauseRehberg et al. ${ }^{17}$ The extended bandgap scheme has been shown ${ }^{37}$ to be incorrect for scaled results, so these levels are not expected to lie inside the experimental bandgap at all. If such a transfer level exists then it most likely originates from a defect complex, rather than an isolated vacancy. This is a possibility that the experiment was not able to exclude.

In conclusion, a comprehensive theoretical overview of the native defects in GaP has been presented. For a wide range of physical circumstances this provides a means to link the different experiments, performed under various different conditions, to an overall picture. It has been shown that the concentrations of grown-in native defects are very sensitive to the stoichiometry at which the GaP sample is grown and also to the Fermi-level position and hence to the degree of doping. The most common native defects have been found to be $\mathrm{P}_{\mathrm{Ga}}^{+2}$ in $p$-type and $\mathrm{Ga}_{\mathrm{P}}^{-2}$ and $\mathrm{V}_{\mathrm{Ga}}^{-3}$ in $n$-type stoichiometric GaP. $\mathrm{P}_{\mathrm{Ga}}^{+2}$ and $\mathrm{V}_{\mathrm{Ga}}^{-3}$ are also the dominant native defects in the $p$-type and $n$-type regions in $\mathrm{P}$-rich $\mathrm{GaP}$, although they are more favorable there than in stoichiometric material. In $\mathrm{GaP}$ grown under Ga-rich conditions $\mathrm{Ga}_{\mathrm{P}}^{-2}$ will be the defect occurring in the highest concentration in semi-insulating material and $n$-type material.
${ }^{1}$ U. Kaufmann, J. Schneider, and A. Räuber, Appl. Phys. Lett. 29, 312 (1976).

${ }^{2}$ T. A. Kennedy and N. D. Wilsey, Phys. Rev. Lett. 41, 977 (1978).

${ }^{3}$ U. Kaufmann, J. Schneider, R. Wörner, T. A. Kennedy, and N. D. Wilsey, J. Phys. C 14, 951 (1981).

${ }^{4}$ T. A. Kennedy and N. D. Wilsey, Phys. Rev. B 23, 6585 (1981).

${ }^{5}$ T. A. Kennedy, N. D. Wilsey, J. J. Krebs, and G. H. Stauss, Phys. Rev. Lett. 50, 1281 (1983).

${ }^{6}$ M. Palczewska, J. Jasinki, K. Korona, M. Kaminska, E. D. Bourret, and A. G. Elliot, J. Appl. Phys. 78, 3680 (1995).

${ }^{7}$ S. Wei, Comput. Mater. Sci. 30, 337 (2004).

${ }^{8}$ P. M. Mooney and T. A. Kennedy, J. Phys. C 17, 6277 (1984).

${ }^{9}$ M. A. Zaidi, M. Zazoui, and J. C. Bourgoin, J. Appl. Phys. 74, 4948 (1993).

${ }^{10}$ T. Neffati and J. C. Bourgoin, Phys. Rev. B 55, 9564 (1997).

${ }^{11}$ X.-Z. Yang, L. Samuelson, H. G. Grimmeiss, and P. Omling, Semicond. Sci. Technol. 3, 488 (1988).

${ }^{12}$ N. Killoran, B. C. Cavenett, M. Godlewski, T. A. Kennedy, and
N. D. Wilsey, J. Phys. C 15, 723 (1982).

${ }^{13}$ B. K. Meyer, Th. Hangleiter, J.-M. Spaeth, G. Struch, Th. Zell, A. Winnacker, and R. H. Bartram, J. Phys. C 18, 1503 (1985).

${ }^{14}$ W. M. Chen and B. Monemar, Phys. Rev. B 40, R1365 (1989).

${ }^{15}$ K. P. O'Donell, K. M. Lee, and G. D. Watkins, Solid State Commun. 44, 1015 (1982).

${ }^{16}$ G. Dlubek, O. Brümmer, and A. Polity, Appl. Phys. Lett. 49, 385 (1986).

${ }^{17}$ R. Krause-Rehberg, A. Polity, W. Siegel, and G. Kühnel, Semicond. Sci. Technol. 8, 290 (1993).

${ }^{18}$ H. Xu, J. Appl. Phys. 68, 4077 (1990).

${ }^{19}$ W. Pötz and D. K. Ferry, Phys. Rev. B 29, 5687 (1984).

${ }^{20}$ W. Pötz and D. K. Ferry, Phys. Rev. B 31, 968 (1985).

${ }^{21}$ P. J. Lin-Chung and T. L. Reinecke, Phys. Rev. B 27, 1101 (1983).

${ }^{22}$ G. S. Khoo and C. K. Ong, Phys. Rev. B 47, 9346 (1993).

${ }^{23}$ G. Schwarz, A. Kley, J. Neugebauer, and M. Scheffler, Phys. Rev. B 58, 1392 (1998). 
${ }^{24}$ San-Guo Shen and Xi-Qing Fan, Phys. Rev. B 49, 14315 (1994).

${ }^{25}$ R. W. Jansen and O. F. Sankey, Phys. Rev. B 39, 3192 (1989).

${ }^{26}$ G. Kresse and J. Furthmuller, Comput. Mater. Sci. 6, 15 (1996).

${ }^{27}$ W. Kohn and L. Sham, Phys. Rev. 140, A1133 (1965).

${ }^{28}$ D. Vanderbilt, Phys. Rev. B 41, R7892 (1990).

${ }^{29}$ D. M. Ceperley and B. J. Alder, Phys. Rev. Lett. 45, 566 (1980).

${ }^{30}$ J. P. Perdew and A. Zunger, Phys. Rev. B 23, 5048 (1981).

${ }^{31}$ D. J. Chadi and M. L. Cohen, Phys. Rev. B 8, 5747 (1973).

${ }^{32}$ H. J. Monkhorst and J. D. Pack, Phys. Rev. B 13, 5188 (1976).

${ }^{33}$ C. G. Van de Walle and J. Neugebauer, J. Appl. Phys. 95, 3851 (2004).

${ }^{34}$ P. E. Blöchl, E. Smargiassi, R. Car, D. B. Laks, W. Andreoni, and S. T. Pantelides, Phys. Rev. Lett. 70, 2435 (1993).

${ }^{35}$ D. B. Laks, C. G. Van de Walle, G. F. Neumark, P. E. Blöchl, and S. T. Pantelides, Phys. Rev. B 45, 10965 (1992).

${ }^{36}$ I. Vurgaftman and J. R. Meyer, J. Appl. Phys. 89, 5815 (2001).
${ }^{37}$ C. W. M. Castleton, A. Höglund, and S. Mirbt (unpublished).

${ }^{38}$ C. W. M. Castleton and S. Mirbt, Phys. Rev. B 68, 085203 (2003).

${ }^{39}$ M. J. Puska, J. Phys.: Condens. Matter 1, 7347 (1989).

${ }^{40}$ M. Scheffler, S. T. Pantelides, N. O. Lipari, and J. Bernholc, Phys. Rev. Lett. 47, 413 (1981).

${ }^{41}$ L. Wang, J. A. Wolk, L. Hsu, E. E. Haller, J. W. Erickson, M. Cardona, T. Ruf, J. P. Silveira, and F. Briones, Appl. Phys. Lett. 70, 1831 (1997).

${ }^{42}$ I. P. Vorona, N. Q. Thinh, I. A. Buyanova, W. M. Chen, Y. G. Hong, and C. W. Tu, Physica B 340-342, 466 (2003).

${ }^{43}$ M. Scheffler, J. Bernholc, N. O. Lipari, and S. T. Pantelides, Phys. Rev. B 29, 3269 (1984).

${ }^{44}$ C. W. M. Castleton and S. Mirbt, Physica B 340-342, 407 (2003); Phys. Rev. B 70, 195202 (2004). 